\title{
LncRNA MEG3 promotes endoplasmic reticulum stress and suppresses proliferation and invasion of colorectal carcinoma cells through the MEG3/ miR-103a-3p/PDHB ceRNA pathway
}

\begin{abstract}
Guoqin WANG ${ }^{1, *}$, Qiuwen $\mathrm{YE}^{2, *}$, Shujuan NING ${ }^{3}$, Zhuangqing YANG ${ }^{4}$, Yunlan $\mathrm{CHEN}^{5}$, Lijuan ZHANG ${ }^{6}$, Youguang $\mathrm{HUANG}^{7}, \mathrm{Fujia} \mathrm{XIE}^{8}$, Xi CHENG ${ }^{8}$, Junlin $\mathrm{CHI}^{8}$, Yi $\mathrm{LEI}^{8}$, Rong $\mathrm{GUO}^{9}$, Jiangqiong $\mathrm{HAN}^{10, *}$

${ }^{1}$ Cancer Biotherapy Center, The Third Affiliated Hospital of Kunming Medical University, Yunnan Cancer Hospital, Kunming, Yunnan, China; ${ }^{2}$ Department of Hepatobiliary and Pancreatic Surgery, The Third Affiliated Hospital of Kunming Medical University, Yunnan Cancer Hospital, Kunming, Yunnan, China; ${ }^{3}$ Department of Rehabilitation medicine, The Third People's Hospital of Yunnan Province, Kunming, Yunnan, China; ${ }^{4}$ Department of Breast surgery, The Third Affiliated Hospital of Kunming Medical University, Yunnan Cancer Hospital, Kunming, Yunnan, China; ${ }^{5}$ Cadre Medical Division, The Third Affiliated Hospital of Kunming Medical University, Yunnan Cancer Hospital, Kunming, Yunnan, China; ${ }^{6}$ Department of Palliative Medicine, The Third Affiliated Hospital of Kunming Medical University, Yunnan Cancer Hospital, Kunming, Yunnan, China; ${ }^{7}$ Cancer Institute, The Third Affiliated Hospital of Kunming Medical University, Yunnan Cancer Hospital, Kunming, Yunnan, China; ${ }^{8}$ Department of Gastrointestinal Surgery, First Affiliated Hospital of Kunming Medical University, Kunming, Yunnan, China; ${ }^{9}$ Department of General Surgery, The Fifth Affiliated Hospital of Kunming Medical University, Kunming, Yunnan, China; ${ }^{10}$ Integrated Traditional Chinese and Western Medicine, The Third Affiliated Hospital of Kunming Medical University, Yunnan Cancer Hospital, Kunming, Yunnan, China
\end{abstract}

${ }^{*}$ Correspondence: $13888320324 @ 163 . c o m$

\#Contributed equally to this work.

Received August 13, 2020 / Accepted September 23, 2020

LncRNA maternally expressed gene 3 (MEG3) is a potential prognostic and diagnostic biomarker in colorectal carcinoma (CC). However, its cellular functions and mechanism remain not fully uncovered. Relative expression of MEG3, miRNA (miR)-103a-3p, and pyruvate dehydrogenase E1 subunit beta (PDHB) was detected by RT-qPCR and western blotting. Cell proliferation was measured by CCK-8 assay, colony formation assay, and flow cytometry, as well as xenograft tumor assay. Transwell assay examined cell invasion. Endoplasmic reticulum (ER) stress was evaluated by western blotting. Dual-luciferase reporter assay and RNA immunoprecipitation determined the relationship between miR-103a-3p and MEG3 or PDHB. Expression of MEG3 was downregulated in human CC tumor tissues and cells (SW620 and HCT116), accompanied by higher miR-103a-3p and lower PDHB. Restoring MEG3 suppressed cell viability, colony formation ability, and invasion, arrested cell cycle, and induced apoptosis rate in SW620 and HCT116 cells, as well as promoted expression of ER stress-related proteins (GRP78, ATF6, CHOP, caspase-3, and caspase-9). Furthermore, MEG3 overexpression hindered tumor growth and facilitated ER stress in vivo. Molecularly, miR-103a-3p was a target of MEG3, and further targeted PDHB. Similarly, in function, blocking miR-103a-3p suppressed CC in vitro by affecting proliferation, invasion, and ER stress; in addition, restoring miR-103a-3p partially counteracted the suppressive role of MEG3 in CC cells. MEG3 sponged miR-103a-3p to suppress CC malignancy by inducing ER stress and inhibiting cell proliferation and invasion via upregulating PDHB, suggesting a novel MEG3/miR-103a-3p/PDHB ceRNA pathway.

Key words: MEG3, ER stress, miR-103a-3p, pyruvate dehydrogenase E1 subunit beta (PDHB), colorectal carcinoma

Endoplasmic reticulum (ER) stress, caused by the accumulation of unfolded or misfolded proteins, plays a dual role in tumorigenesis and development [1], by linking to fundamental biological processes, such as proliferation, apoptosis, migration, invasion, and autophagy [2-4]. In response to ER stress, unfolded protein response (UPR) is activated to promote tumor-survival response or anti-tumor response $[5$,
6]. Not surprisingly, ER stress and UPR are associated with intestinal diseases, including colorectal carcinoma (CC) $[7,8]$.

$\mathrm{CC}$ is the third most common cancer according to global cancer statistics 2018 of the International Agency for Research on Cancer [9], and its incidence and mortality are expected to be rapidly increasing in many low-income and middle-income countries [10]. The survival of CC patients 
is closely related to the stage of CC [11], thus it is essential to identify more efficient molecular targets for a better understanding of the mechanism underlying CC progression, as well as seeking early diagnostic markers.

Recently, it has been well documented that long noncoding RNAs (lncRNAs) as key regulators mediate the competing endogenous RNA (ceRNA) network to regulate carcinogenesis, progression, and treatment of CC $[12,13]$. LncRNA maternally expressed gene 3 (MEG3) is the first identified tumor suppressive lncRNA in human cancers. In CC, MEG3 has been suggested as a prognostic biomarker for CC [14, 15]; nevertheless, its role and mechanism in CC cells remain largely unclear, especially in ER stress.

The interactions among lncRNAs, microRNAs (miRNAs), and messenger RNAs (mRNAs) have participated in tumorigenesis, growth, progression, epithelial-mesenchymal transition, drug resistance, and therapeutic potential in CC [16]. Moreover, miRNAs have a dual role for CC progression both in oncogenes and tumor suppression [17]. miRNA (miR)-103a-3p is one of a panel of miRNAs identified as a biomarker for the diagnosis and prognosis of CC $[18,19]$. However, the detailed biological functions of this miRNA are left to be illustrated in CC cells, as well as its underlying regulatory mechanism.

In this study, we focused on investigating the role of MEG3 and miR-103a-3p in proliferation, invasion, and ER stress of CC cells, as well as the corresponding ceRNA mechanism through regulating pyruvate dehydrogenase E1 subunit beta (PDHB), a circadian clock-regulated glycolysis gene that is involved in cancer progression and metastasis [20].

\section{Materials and methods}

Human tissue samples. Human colorectal tumor tissues and adjacent normal colorectal mucosal tissues were obtained from a group of 33 patients with CC at the Third Affiliated Hospital of Kunming Medical University. The patients received no antitumor therapies before this surgery, and CC pathological classification was applied in conformity to the Union for International Cancer Control (UICC) CC staging criteria. All tissues were harvested after each patient signed written consent for further RNA and protein isolation. This

Table 1. Sequences of primers used in the study.

\begin{tabular}{ll}
\hline Gene & Primers \\
\hline MEG3 & $\begin{array}{l}\text { forward 5'-CCACCCCTCTTGCTTGTCTT-3' } \\
\text { reverse 5'-GGCCTTTCAAGAAGCTTGGC-3' } \\
\text { forward 5'-CAGCATTGTACAGGGCT-3' } \\
\text { reverse 5'-GAACATGTCTGCGTATCTC-3' } \\
\text { forward 5'-CGGATAGAGGACACGACCAAG-3' } \\
\text { PDHB }\end{array}$ \\
reverse 5'-TGAAAGCGCCTCTTCAGCA-3' \\
GAPDH & $\begin{array}{l}\text { revward 5'-CCACATCGCTCAGACACCAT-3' } \\
\text { forward 5'-ACCAGGCGCCCAATACG-3' } \\
\text { reverse 5'-CGCTTCACGACATTTGCGTGTCAT-3' }\end{array}$ \\
\hline
\end{tabular}

work was approved by the Ethics Committee of the Third Affiliated Hospital of Kunming Medical University.

Cells and cell culture. Human normal colonic mucosal epithelial cell line FHC (CRL-1831), human embryonic kidney epithelial cell line 293T (CRL-3216), and human CC cell lines SW620 (CCL-227) and HCT116 (CCL-247) were from the American Type Culture Collection (ATCC; Manassas, VA, USA). These cells were cultured in corresponding mediums according to culture methods on ATCC. FHC, 293T, and HCT116 cells were maintained at $37^{\circ} \mathrm{C}$ in a humidified atmosphere containing $5 \% \mathrm{CO}_{2}$, while SW620 cells were cultivated without $\mathrm{CO}_{2}$.

Real-time quantitative PCR (RT-qPCR). Total RNA was isolated using TRIzol reagent (Invitrogen, Carlsbad, CA, USA), followed by the identification of the quality and concentration on Nanodrop 2000 spectrophotometer (Thermo Fisher Scientific, Foster City, CA, USA). An aliquot of RNA (500 ng) was subjected to cDNA synthesis and amplification using the RevertAid First-Strand cDNA Synthesis kit (Thermo Fisher Scientific) and SYBR Green Mix (Thermo Fisher Scientific), respectively. Glyceraldehyde-phosphate dehydrogenase (GAPDH) was used for the normalization of MEG3 and PDHB, while U6 small nuclear RNA (U6) was for miR-103a-3p. The primers' sequences are shown in Table 1. The relative expression level was calculated using the $2^{(-\Delta \Delta(T))}$ method.

Cell transfection. The overexpressing vector pcDNA3.1 (+) (vector; Invitrogen) was used to construct the MEG3 overexpression vector (oe-MEG3) by inserting the full length of MEG3 (NR_002766). The mimics of miR-103a-3p and negative control (miR-103a-3p mimic and mimic NC), inhibitors of miR-103a-3p and negative control (miR-103a-3p inhibitor and inhibitor NC), and siRNAs against PDHB and negative control (si-PDHB and si-NC) were separately synthesized by Songon Biotech (Shanghai, China). In general, these nucleotides were transfected into SW620 and HCT116 cells using Lipofectamine 3000 reagent (Invitrogen) according to the manufacturers' instruction. The doses of nucleotides in transfection are summarized in Supplementary Table S1. For co-transfection, nucleotides were mixed for transfection with and two-fold of Lipofectamine 3000 reagent (Invitrogen). The siRNAs sequence was listed: si-PDHB sense 5'-GGAAUUCAGAGGAUGCUAAAG-3' and antisense 5'-UUAGCAUCCUCUGAAUUCCAG-3', and si-NC sense 5'-GCUACGAUCUGCCCAAGAUTT-3' and antisense 5'-AUCUUAGGCAFGAUCGUCGCTT-3'.

Cell counting kit (CCK)-8 assay, colony formation assay, and cell cycle analysis. For monitoring cell viability, SW620 and HCT116 cells were plated into 96-well plates with different transfection. After transfection for $24 \mathrm{~h}, 48 \mathrm{~h}$, and $72 \mathrm{~h}, 10 \mu \mathrm{l}$ of CCK-8 solution (Dojindo, Tokyo, Japan) was added into each well ( 5 wells per group). After further incubation for $2 \mathrm{~h}$, the optical density (OD) values of cells were measured at $450 \mathrm{~nm}$ on the microwell plate spectrophotometer. 
For colony formation assay, transfected SW620 and HCT116 cells in 6-well plate were transferred into a fresh a 6 -well plate with 400 cells/well (3 wells/group). After further cell culturing for 15 days, cell colonies were formed and counted with crystal violet staining. The number of stained colonies was calculated under a microscope.

To analyze cell cycle distributions, transfected SW620 and HCT116 cells were fixed with $75 \%$ ethanol overnight at $4{ }^{\circ} \mathrm{C}$ and stained with the cell cycle detection kit (Keygen, Nanjing, China). Lastly, the DNA content was measured on the Accuri C6 flow cytometer (BD Biosciences, San Jose, CA, USA).

Transwell assay. For invasion assay, transwell chambers (8.0 $\mu \mathrm{m}$ pore size; EMD Millipore, Billerica, MA, USA) and Matrigel (Corning, NY, USA) were used. The chambers were prepared with Matrigel ( $10 \%$ in medium) at room temperature for overnight. After that, $5 \times 10^{5}$ cells (SW620 and HCT116) suspended in serum-free medium were placed in the upper chambers (three chambers per group), and medium supplemented with $20 \%$ fetal bovine serum (Hyclone, Logan, UT, USA) was added in the lower chamber. After further cultivation for $48 \mathrm{~h}$, the invaded cells on the lower surface were fixed with ice-cold $75 \%$ ethanol overnight at $4{ }^{\circ} \mathrm{C}$ and stained with $0.2 \%$ crystal violet for $1 \mathrm{~h}$ at room temperature. The number of invaded cells was counted a under microscope $(100 \times)$.

Apoptosis assay. For cell apoptosis assay, double staining method and flow cytometry (FCM) were employed. After transfection, SW620 and HCT116 cells were subjected to Annexin V- fluorescein isothiocyanate (FITC) Apoptosis Detection Kit (Beyotime, Shanghai, China) according to the instructions. Stained cells were sorted in Annexin V/ propidium iodide (PI) quadrant and apoptotic cells were in Annexin V+/PI+ and Annexin V+/PI- quadrants.

Western blotting. The assay was implemented according to the previous description [21]. The primary antibodies used are listed as follows: glucose-regulated protein 78 (GRP78; sc-13539), activating transcription factor 6 (ATF6; sc-166659), C/EBP homologous protein (CHOP; sc-7351), and GAPDH (sc-47724) were from Santa Cruz (Shanghai, China), as well as PDHB (ab155996), cleaved caspase-3 (caspase-3; ab49822) and caspase-9 p35 (caspase-9; ab210611) from Abcam (Cambridge, UK). Protein level was quantified by the Image software (National Institutes of Health, Bethesda, MD, USA) with normalization to GAPDH.

Dual-luciferase reporter assay and RNA immunoprecipitation (RIP) assay. The putative binding sites of miR-103a-3p in wild-type (wt) of MEG3 (MEG3 wt) or 3'UTR of PDHB wt (PDHB 3'UTR wt) were mutated using the Quickchange XL Site-Directed Mutagenesis Kit (Agilent Stratagene, Cedar Creek, Texas, USA) according to the manufacturer's protocol. Then, 293T cells were seeded in a 96-well plate and co-transfected with $50 \mathrm{ng}$ Firefly luciferase reporter vector pGL3 (Promega, Madison, WI, USA) carrying MEG3 wt, PDHB 3'UTR wt, or their mutants (MEG3 mut or PDHB 3'UTR mut), 5 ng Renila luciferase reporter vector pRL-TK (Promega), and miRNA mimic. After transfection for 48 h, Firefly and Renila luciferase activities were detected using the dual-luciferase reporter assay system (Promega), and Renila luciferase activity was the internal control.

For RIP assay, SW620 and HCT116 cells were lysed in RIP lysis buffer, and then cell lysate was subjected to EZ-Magna RIP Kit (Millipore, Billerica, MA, USA) according to the instructions. Antibodies against Argonaute 2 (anti-Ago2; ab32381) or immunoglobulin G (anti-IgG; ab172730) were from Abcam. The RNA expression in RIP portions (Input, IgG-RIP, and Ago2-RIP) was further detected by RT-qPCR.

In vivo experiment. This animal experiment was approved by the Ethics Committee of the Third Affiliated Hospital of Kunming Medical University. Male nude mice (Laboratory Animal Center of Nanjing University, Nanjing, China) were purchased and raised for xenograft tumor models. SW620 cells $\left(5 \times 10^{6}\right)$ stably transfected with oe-MEG3 or empty vector were further subcutaneously seeded into the flanks of the above mice $(n=6)$. The mice were monitored every three days from transplantation, and the dimensions (length and width) of neoplasms were measured using a caliper. All xenograft mice were euthanatized on the last day of the xenograft experiment and the tumor weight was measured using an electronic balance. Tissues of mice tumors were stored for further RNA and protein isolation. The tumor volume was calculated as $0.5 \times$ length $\times$ width $^{2}$. All animal operations strictly followed the Guide for the Care and Use of Laboratory Animals from the National Institutes of Health.

Statistical analysis. Values are presented as mean \pm standard deviation with data analysis on GraphPad Prism version 5.00 (GraphPad Software, San Diego, CA, USA). Comparisons were analyzed via Student's t-test or analysis of variance for experiments with two groups or multiple groups. The correlation between miR-103a-3p and MEG3 or PDHB mRNA levels was determined by Pearson's correlation analysis. A p-value $<0.05$ was deemed as a statistical difference.

\section{Results}

MEG3 expression was decreased in CC patients and cells. Expression status of MEG3 in CC was confirmed in a cohort of patients with CC, and RT-qPCR data showed a lower level of MEG3 in tumor tissues (0.81-fold) than adjacent normal tissues (1.46-fold) (Figure 1A). Besides, the MEG3 expression was also decreased in human CC cell lines including SW620 (0.30-fold) and HCT116 (0.43-fold), in parallel with normal FHC cells (1.0-fold) (Figure 1B). These outcomes demonstrated the downregulation of MEG3 in CC, suggesting a potential tumor-suppressive role of MEG3.

Boosting MEG3 restrained CC cell proliferation and invasion, and promoted ER stress in vitro. The inherent downregulation of MEG3 was artificially restored using oe-MEG3 transfection, and RT-qPCR revealed a significant increase of the MEG3 expression in SW620 and HCT116 cells transfected with oe-MEG3 (Figure 2A). Subsequently, the 

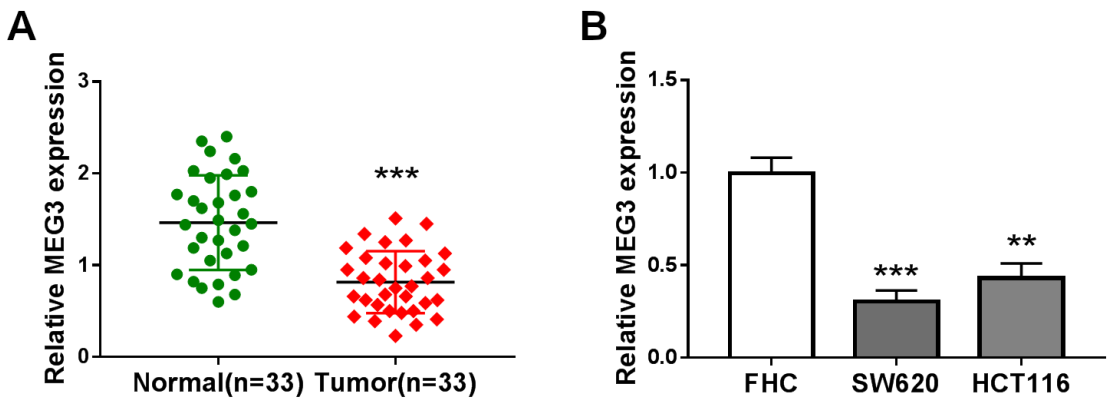

Figure 1. Detecting the expression of lncRNA maternally expressed gene 3 (MEG3) in human colorectal carcinoma (CC) tissues and cells. RT-qPCR detected the relative MEG3 expression in A) tumor tissues and adjacent normal tissues from patients with CC (n=33), and B) CC cell lines SW620 and HCT116, as well as normal colonic mucosal cell line FHC. ${ }^{* *} \mathrm{p}<0.01$ and ${ }^{* * *} \mathrm{p}<0.001$.

role of MEG3 dysregulation in CC cells was figured out. The CCK-8 assay indicated that the cell viability of SW620 and HCT116 cells was inhibited after oe-MEG3 transfection, as displayed by lowered OD values at $48 \mathrm{~h}$ and $72 \mathrm{~h}$ (Figure $2 \mathrm{~B}$ ). Colony formation assay manifested a loss of colonies formed in oe-MEG3-transfected SW620 and HCT116 cells (Figure 2C). FCM data demonstrated that oe-MEG3 transfection rendered cell cycle arrest in SW620 and HCT116 cells, as evidenced by increased the G1/G0 phase cells and decreased $S$ phase cells (Figure 2D). The above results illuminated that a proliferation inhibition was mediated by MEG3 overexpression in CC cells in vitro.

Next, the invasion of SW620 and HCT116 cells was measured by transwell assay, and invaded cells were descended due to the oe-MEG3 transfection (Figure 2E). Besides, apoptosis was promoted in SW620 and HCT116 cells with MEG3 restoration, as certified by a higher apoptosis rate (Figure 2F) and elevated expression of caspase- 3 and caspase-9 (Figure 2G). Western blotting results indicated that UPR response-related proteins, including GRP78, ATF6, and CHOP [22], were highly induced by restoring MEG3 (Figure $2 \mathrm{G}$ ). These outcomes also expounded that invasion inhibition and ER stress promotion were mediated by the MEG3 overexpression in CC cells in vitro.

MEG3 knockdown regulated cell proliferation, invasion, and ER stress of CC cells through functioning as a sponge of miR-103a-3p. The miRcode software (http://mircode. org $/$ ? gene $=$ MEG3 \&mirfam $=$ \&class $=\&$ cons $=$ \&trregion $=$ ) was utilized to predict the target miRNAs of MEG3. Among the multiple miRNAs, the expression of miR-103a-3p was upregulated in tumor tissues (2.42-fold) from CC patients (Figure 3A), as well as CC cell lines SW620 and HCT116 (2.19-fold and 1.92-fold) (Figure 3B); moreover, its expression was negatively correlated with MEG3 in CC tumors (Figure 3C). Thus, miR-103a-3p was selected for further identification, and the potential binding sites between miR-103a-3p and MEG3 wt are presented in Figure 3D. Dual-luciferase reporter assay revealed a decline of luciferase activity in 293T cells transfected with MEG3 wt combined with miR-103a-3p mimic than with mimic NC (Figure 3E) and MEG3 mut groups showed no significant difference on luciferase activity. RIP assay revealed the enrichment of both MEG3 and miR-103a-3p in the cell lysate of SW620 and HCT116 cells incubated with anti-Ago2 (Figure 3F). These results testified to the direct interaction between MEG3 and miR-103a-3p.

In functions, the contribution of miR-103a-3p to the role of MEG3 in proliferation, invasion, and ER stress was further detected in rescue experiments. SW620 and HCT116 cells underwent the transfection of oe-MEG3 alone or together with miRNA mimics. miR-103a-3p was monitored for the inhibition with oe-MEG3 transfection, and this downregulation was rescued with miR-103a-3p mimic co-transfection (Figure 3G). The cell viability suppression in oe-MEG3transfected SW620 and HCT116 cells was attenuated in the presence of miR-103a-3p mimic (Figure $3 \mathrm{H}$ ), as well as suppression on colony formation ability and promotion on cell cycle arrest in the G1/G0 phase (Figures 3I, 3J, and Supplementary Figure S1A). The cell invasion was also inhibited in SW620 and HCT116 cells with the oe-MEG3transfection, which was improved with the co-transfection of miR-103a-3p mimic (Figure 3K and Supplementary Figure $\mathrm{S} 1 \mathrm{~B})$. Introducing oe-MEG3-induced ER stress was mitigated by the miR-103a-3p mimic administration, as described by a reduced apoptosis rate (Figure $3 \mathrm{~L}$ and Supplementary Figure S1C), and lowered expression of GRP78, ATF6, CHOP, caspase-3, and caspase- 9 (Figure 3M). These results further confirmed the interactive effect between MEG3 and miR-103a-3p in functions, as well.

$\mathrm{PDHB}$ as one target of miR-103a-3p was downregulated in human CC tumor tissues and cells. The Targetscan software (http://www.targetscan/PDHB ENST00000474765.1\&taxid=miR-103a-3p) showed PDHB as a potential target of miR-103a-3p, and PDHB expression both mRNA level was downregulated in human CC tumor tissues (1.07-fold) than adjacent tissues (1.96-fold), as well as CC cell lines ( 0.31 fold and 0.43 fold) versus FHC ( 1.00 -fold) (Figures $4 \mathrm{~A}$ and $4 \mathrm{C}$ ). Similarly, PDHB protein expression in 
A

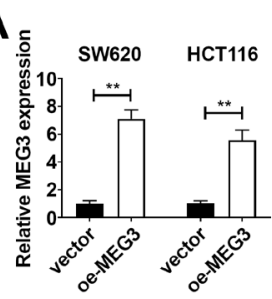

B
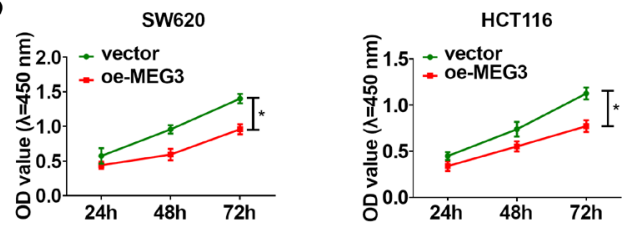

C

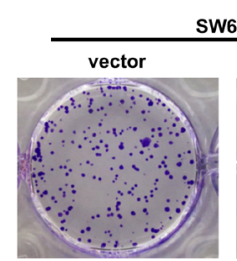

SW620
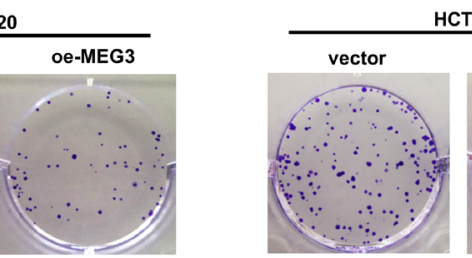

HCT116
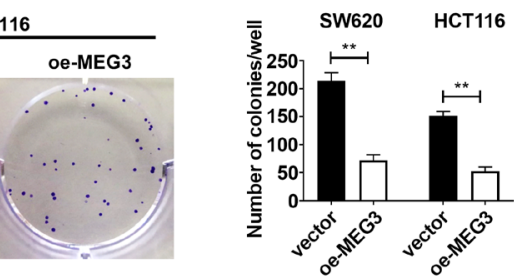

D
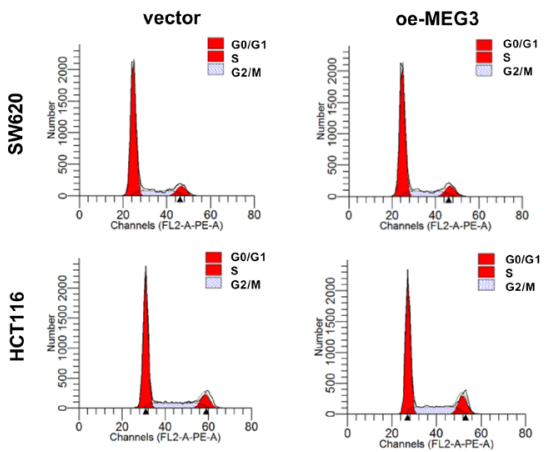

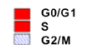
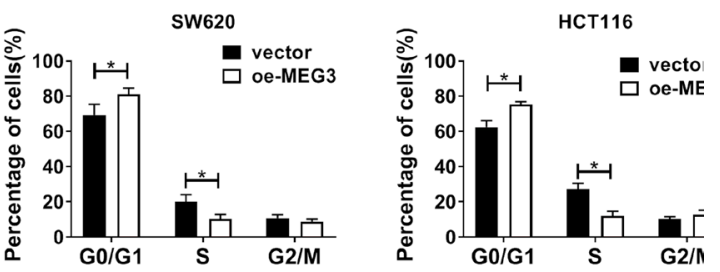

E

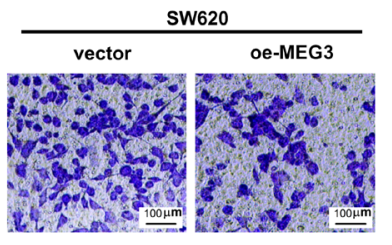

F SW620
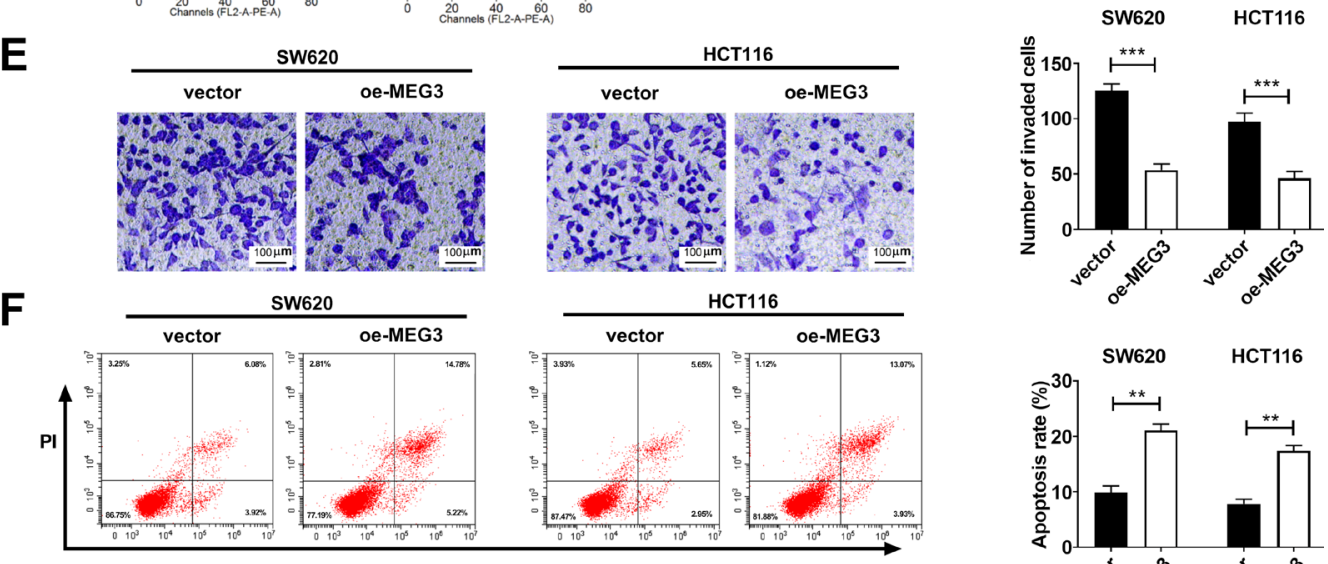

\section{G}

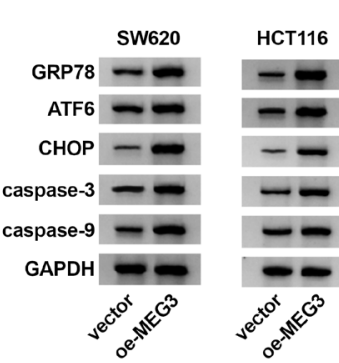

Annexin V-FITC
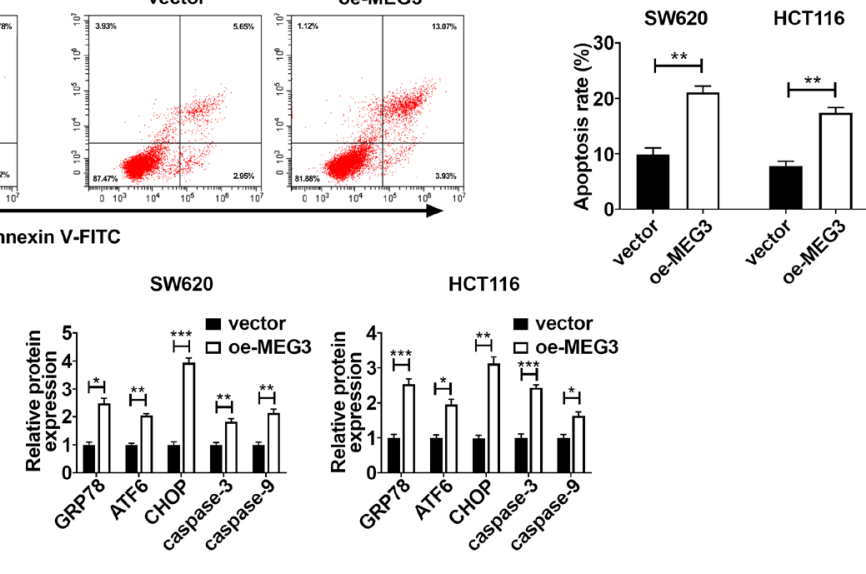

Figure 2. Exploring the effects of MEG3 on cell proliferation, invasion, and endoplasmic reticulum (ER) stress of CC cells in vitro. A-G) SW620 and HCT116 cells underwent the transfections of MEG3 overexpressing vector (oe-MEG3) and empty vector (vector), respectively. After transfection, (A) RT-qPCR detected the relative MEG3 expression, (B) CCK-8 measured the optical density (OD) value at $450 \mathrm{~nm}$, (C) the colony formation assay examined the number of colonies formed, (D) flow cytometry (FCM) determined the percentage of cells in different cell cycle phases, (E) the transwell assay evaluated the number of invaded cells, (F) FCM determined the apoptosis rate, and (G) western blotting tested the relative protein expression of ER stress-related proteins, including glucose regulated protein 78 (GRP78), activating transcription factor 6 (ATF6), C/EBP homologous protein (CHOP), cleaved caspase-3 (caspase-3), and cleaved caspase-9 (caspase-9). ${ }^{*} \mathrm{p}<0.05,{ }^{* *} \mathrm{p}<0.01$, and ${ }^{\star * *} \mathrm{p}<0.001$. 
A

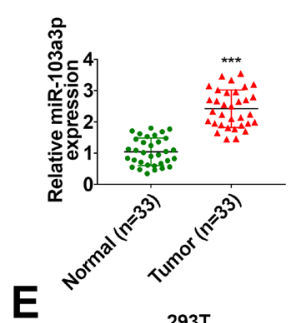

B

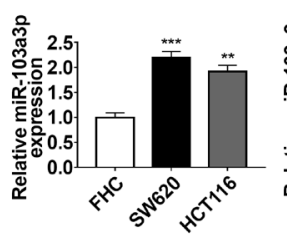

F sw620
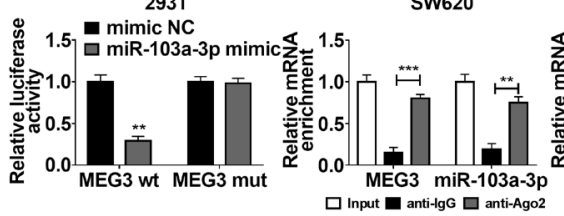

C

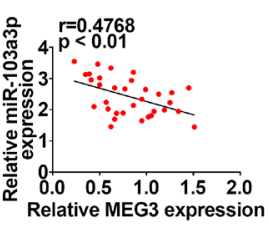

D

\author{
MEG3 wt 5'-GTTAGTGCTGCA-3' \\ miR-103a-3p 3'-ATGTTACGACGA-5' \\ MEG3 mut 5'-GTTAGACGACGA-3'
}
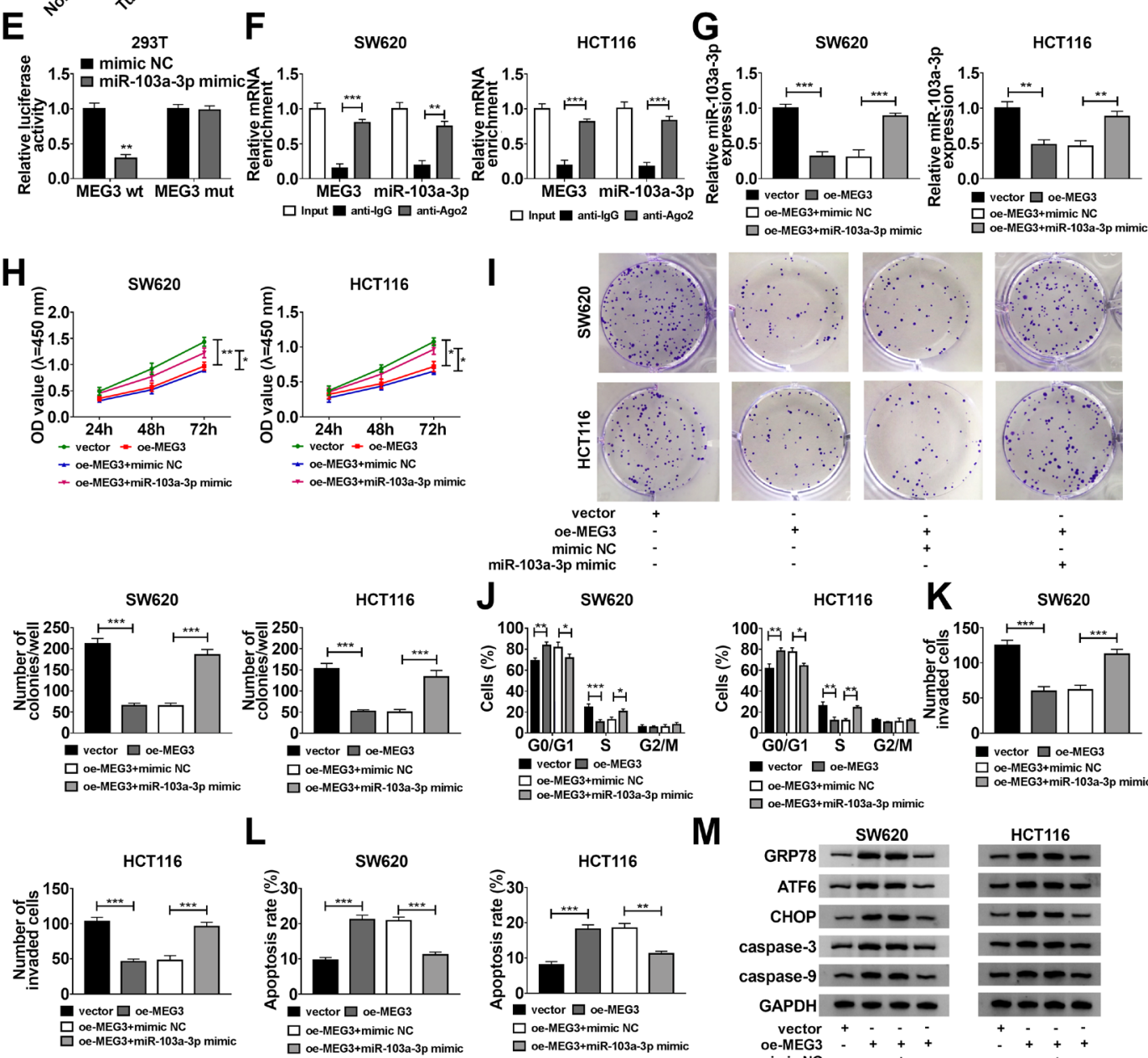

M
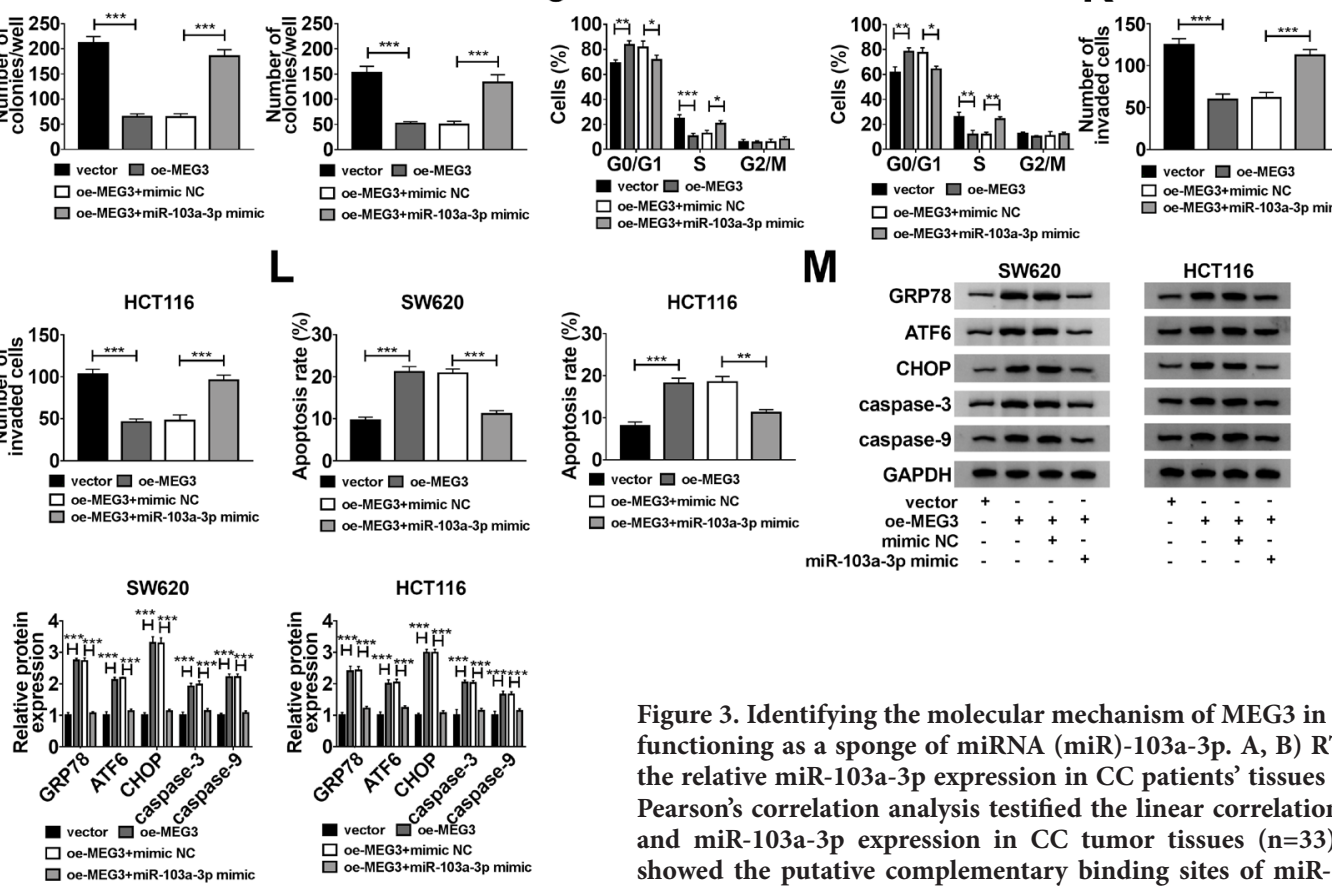

Figure 3. Identifying the molecular mechanism of MEG3 in CC cells through functioning as a sponge of miRNA (miR)-103a-3p. A, B) RT-qPCR detected the relative miR-103a-3p expression in CC patients' tissues and cell lines. C) Pearson's correlation analysis testified the linear correlation between MEG3 and miR-103a-3p expression in CC tumor tissues $(n=33)$. D) The sketch showed the putative complementary binding sites of miR-103a-3p in wildtype of MEG3 (MEG3 wt). E) Dual-luciferase reporter assay measured the relative luciferase activity of MEG3 wt and its mutant (MEG3 mut) in 293T cells transfected with mimics of miR-103a-3p or its negative control (miR-103a-3p mimic or mimic NC). F) RNA immunoprecipitation assay detected the relative enrichment of MEG3 and miR-103a-3p in the input of cell lysate (Input) incubated with antibody against immunoglobulin G (anti-IgG) or antibody against Argonaute 2 (anti-Ago2). G-M) SW620 and HCT116 cells underwent transfections of vector, oe-MEG3, oe-MEG3 combined with mimic NC, and oe-MEG3 combined with miR-103a-3p. (G) RT-qPCR detected the relative miR-103a-3p expression. (H) CCK-8 assay measured the $O D$ value at $450 \mathrm{~nm}$. (I) The colony formation assay examined the number of colonies formed. (J) FCM determined the percentage of cells in different cell cycle phases. (K) The transwell assay evaluated the number of invaded cells. (L) FCM determined the apoptosis rate. (M) Western blotting tested the relative protein expression of GRP78, ATF6, CHOP, caspase-3, and caspase-9. ${ }^{*} \mathrm{p}<0.05,{ }^{* *} \mathrm{p}<0.01$, and ${ }^{* * *} \mathrm{p}<0.001$. 
CC patients and cells was consistent with its mRNA expression (Figures 4B and 4D). Moreover, there was an inverse correlation between miR-103a-3p and PDHB mRNA expression in CC patients' tumors (Figure 4E). According to the predicted binding sites of miR-103a-3p in PDHB 3'UTR wt (Figure 4F), PDHB 3'UTR mut was established for further dual-luciferase reporter assay. As a result, only luciferase activities of PDHB 3'UTR wt was reduced in 293T cells co-transfected with miR-103a-3p mimic (Figure 4G). RIP assay also revealed the enrichment of both miR-103a-3p and PDHB in the cell lysate of SW620 and HCT116 cells incubated with anti-Ago2 (Figure 4H). These data suggested $\mathrm{PDHB}$ as a downstream target of miR-103a-3p.

Blocking miR-103a-3p upregulated PDHB to suppress $\mathrm{CC}$ in vitro via inhibiting cell proliferation and invasion, and enhancing ER stress. Herewith, the mutual effect of miR-103a-3p and PDHB was then testified. The transfection of miR-103a-3p inhibitor led to the downregulation of miR-103a-3p in SW620 and HCT116 cells (Figure 5A), accompanied by the upregulation of PDHB mRNA expression and protein expression (Figures 5B, 5C). In addition, higher PDHB expression mediated by miR-103a-3p silencing was further attenuated with si-PDHB transfection (Figures 5B, 5C). OD values of SW620 and HCT 116 cells were lowered after miR-103a-3p inhibitor transfection at $48 \mathrm{~h}$ and $72 \mathrm{~h}$, suggesting a cell viability inhibition (Figure 5D). The number of colonies formed was declined in miR-103a-3p inhibitortransfected SW620 and HCT116 cells (Figure 5E), indicating suppression of colony formation ability. miR-103a-3p inhibitor transfection arrested cell cycle in SW620 and HCT116 cells, as evidenced by increased G1/G0 phase cells and decreased S phase cells (Figure 5F and Supplementary Figure S2A). Cell invasion of SW620 and HCT116 cells was depressed due to the miR-103a-3p inhibitor transfection, as depicted by the descended number of invaded cells (Figure 5G and Supplementary Figure S2B). Besides, the apoptosis rate and
A

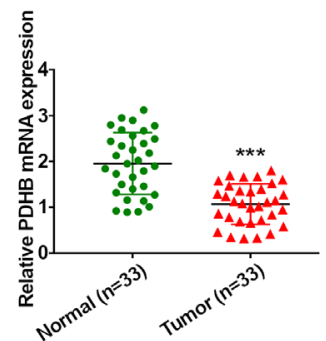

E

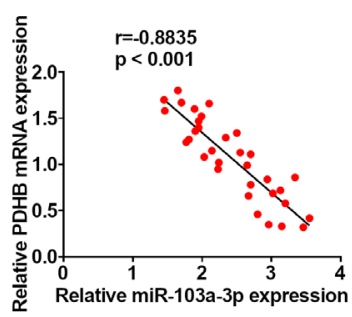

$\mathrm{H}$

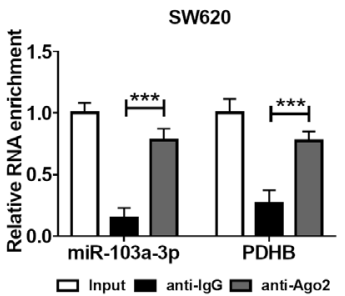

B

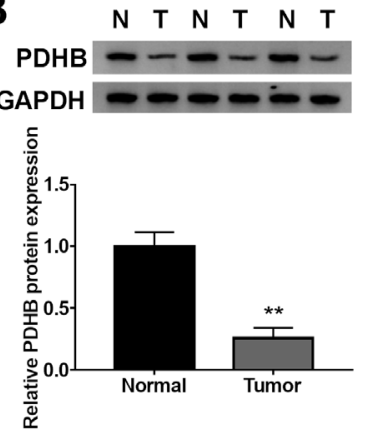

$\mathbf{F}$

miR-103a-3p

5'-UgaAaAuAuUUCAAUAUgCUgCU-3' 3'-ACUAUCGgGACAUGUUACGACGA-5'

PDHB 3'UTR mut 5'-UGAAAAUAUUUCAAUUACGACGU-3'
D

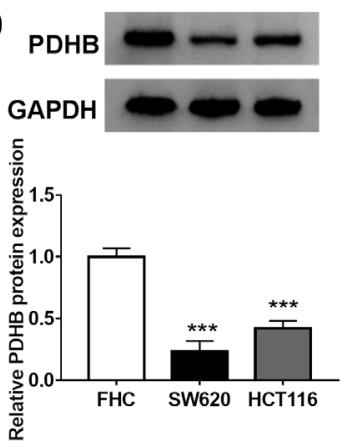

G

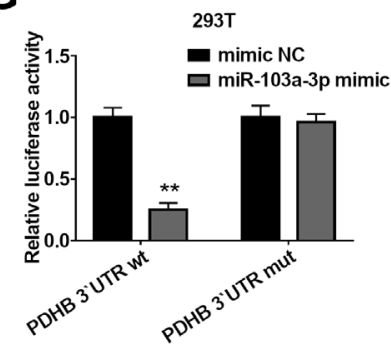

HCT116

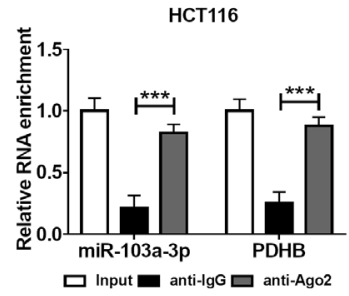

Figure 4. Identification of pyruvate dehydrogenase E1 subunit beta (PDHB) as a downstream target of miR-103a-3p in CC cells. RT-qPCR and western blotting detected the relative PDHB mRNA expression and protein expression in (A, B) tissues from patients with CC ( $\mathrm{n}=33$ ), and $(\mathrm{C}$, D) cell lines of FHC, SW620, and HCT116. E) Pearson's correlation analysis testified the linear correlation between miR-103a-3p and PDHB mRNA expression in CC tumor tissues $(n=33)$. F) The sketch showed the putative complementary binding sites of miR-103a-3p in the wild-type of 3'UTR of PDHB (PDHB 3'UTR wt). G) Dual-luciferase reporter assay measured the relative luciferase activity of PDHB 3'UTR wt and its mutant (PDHB 3'UTR mut) in 293T cells transfected with miR-103a-3p mimic or mimic NC. (H) RIP assay validated the RNA enrichment of miR-130a-3p and PDHB in Input incubated with anti-IgG or anti-Ago2. ${ }^{* *} \mathrm{p}<0.01$ and ${ }^{\star * *} \mathrm{p}<0.001$. 
A

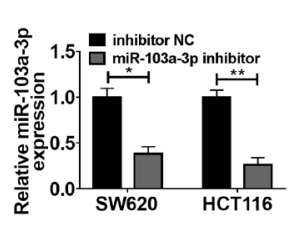

D
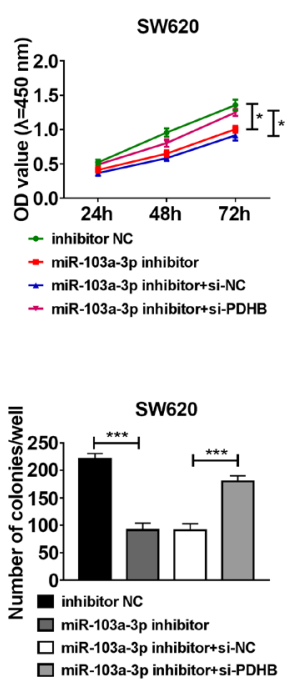

G
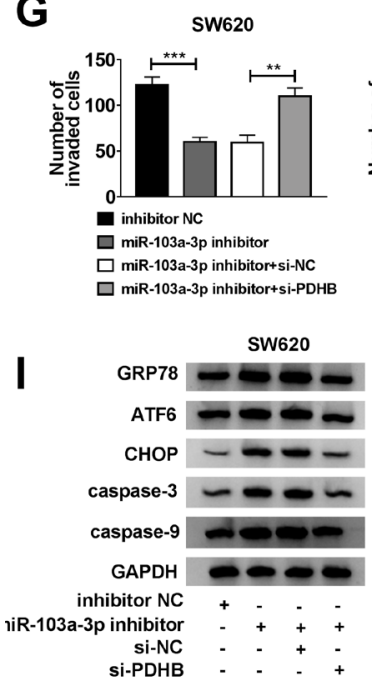

B
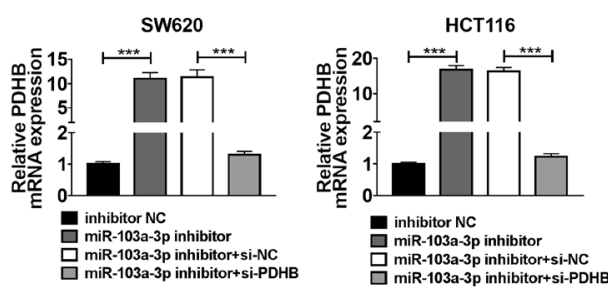

E
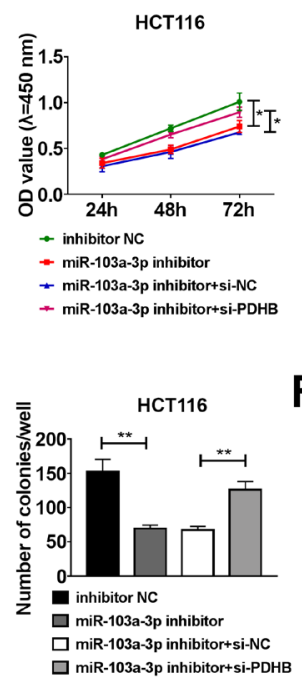

$\mathbf{F}$ inhibitor NC
miR-103a-3p inhibitor
si-NC si-NC
si-PDHB
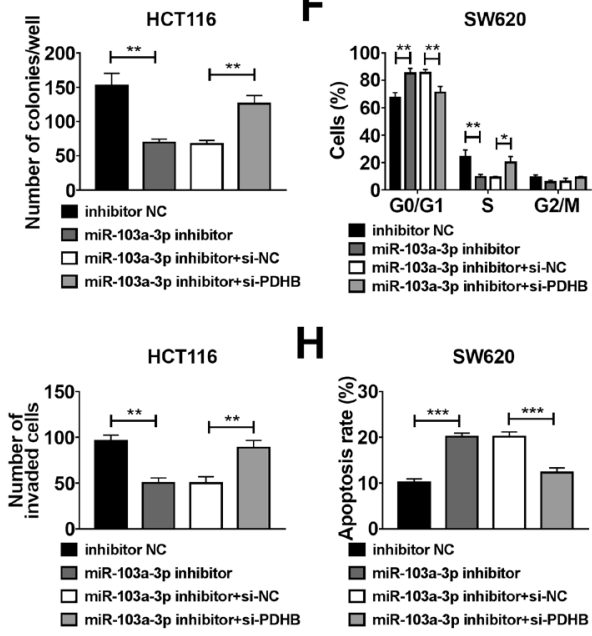

$\mathbf{H}$
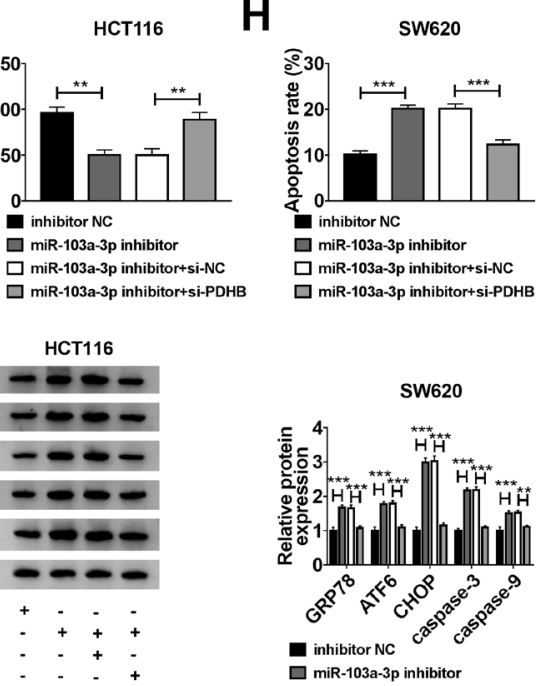

C

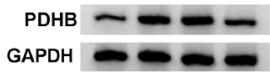

$\mathrm{PDHB}-0-$ GAPDH 0
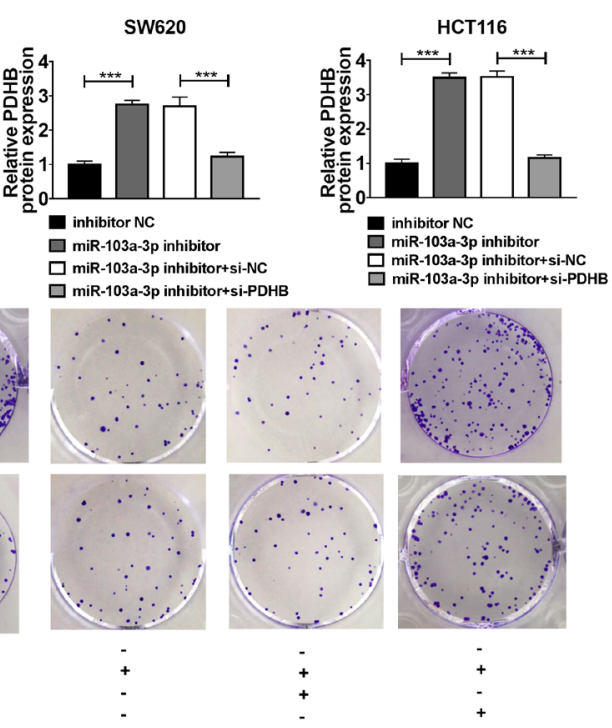

HCT116
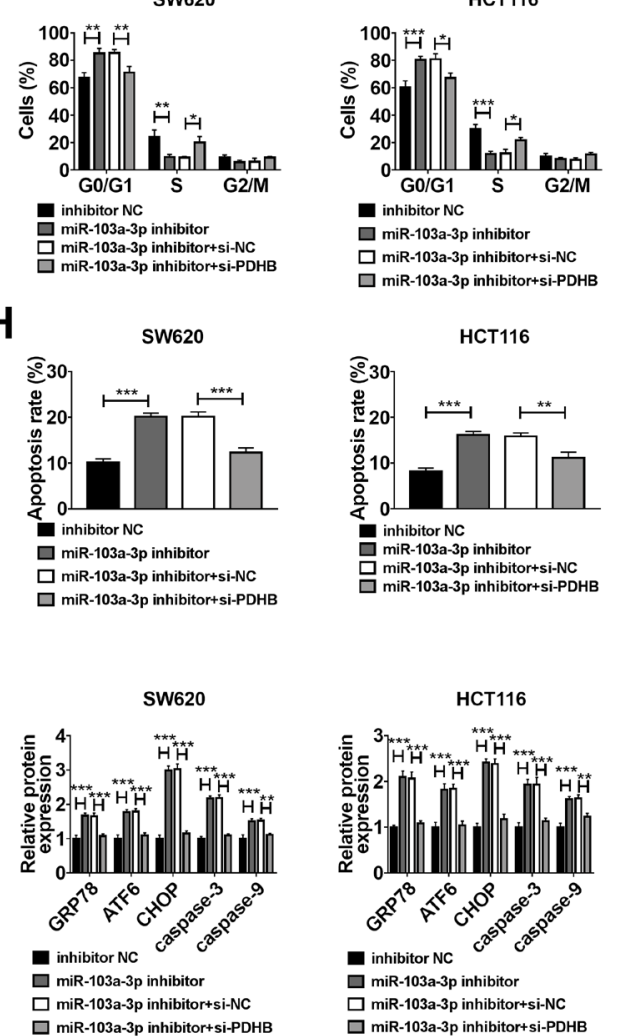

Figure 5. Identifying the mutual effect of miR-103a-3p and PDHB on cell proliferation, invasion, and ER stress of CC cells in vitro. A) RT-qPCR detected the relative miR-103a-3p expression in SW620 and HCT116 cells transfected with the inhibitors of miR-103a-3p or the negative control (miR103a-3p inhibitor or inhibitor NC). B-I) SW620 and HCT116 cells underwent transfections of inhibitor NC, miR-103a-3p inhibitor, and miR-103a-3p inhibitor combined with siRNAs target PDHB or the negative control (si-PDHB or si-NC). (B, C) RT-qPCR and western blotting detected the relative PDHB mRNA and protein expression. (D) CCK-8 measured the OD value at $450 \mathrm{~nm}$. (E) The colony formation assay examined the number of colonies formed. (F) FCM determined the percentage of cells in different cell cycle phases. (G) The transwell assay evaluated the number of invaded cells. (H) FCM determined the apoptosis rate. (I) Western blotting tested the relative protein expression of GRP78, ATF6, CHOP, caspase-3, and caspase-9. ${ }^{\star} \mathrm{p}<0.05,{ }^{\star *} \mathrm{p}<0.01$, and ${ }^{\star * *} \mathrm{p}<0.001$. 
expression of caspase-3, caspase-9, GRP78, ATF6, and CHOP were consistently promoted in SW620 and HCT116 cells with the miR-103a-3p deletion (Figures 5H, 5I, and Supplementary Figure S2C), hinting an induction of ER stress and the activation of the ATF6-mediated UPR pathway. These outcomes together demonstrated that silencing miR-103a-3p suppressed proliferation and invasion, but promoted ER stress in CC cells in vitro. By the co-transfection with si-PDHB, the effects of miR-103a-3p knockdown via inhibitor transfection were partially overturned in both SW620 and HCT116 cells (Figures 5D-5I). This outcome manifested that the miR-103a-3p inhibition upregulated PDHB to play a suppressive role in CC cells in vitro.
MEG3 upregulated the PDHB expression in CC cells via downregulating miR-103a-3p. And then, we observed that PDHB mRNA and protein expression levels were high in SW620 and HCT116 cells with MEG3 overexpression, and this upregulation was also abrogated by the transfection of miR-103a-3p mimic (Figures 6A-6D). This phenomenon implied that MEG3 served as a ceRNA of miR-103a-3p to positively modulate PDHB.

Overexpressing MEG3 hindered tumor growth and induced ER stress in CC cells in vivo. Since a tumor-suppressive role of MEG3 in CC cells in vitro had been confirmed by regulating proliferation, invasion, and ER stress, its role in vivo was next investigated. Xenograft tumors were gener-
A

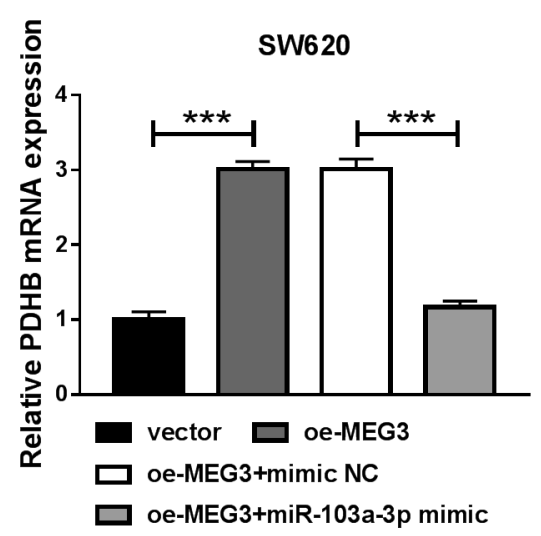

C

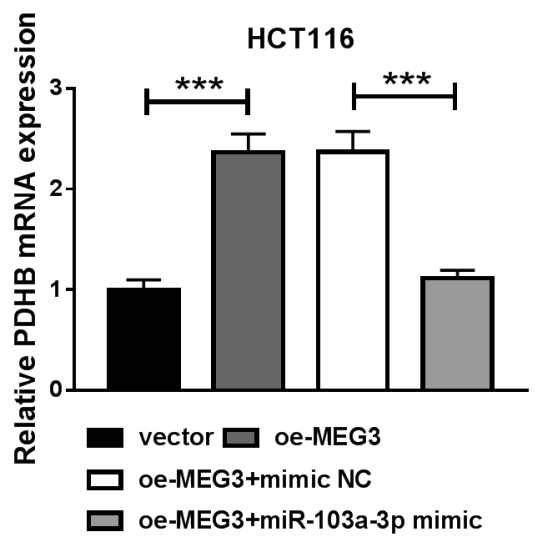

B
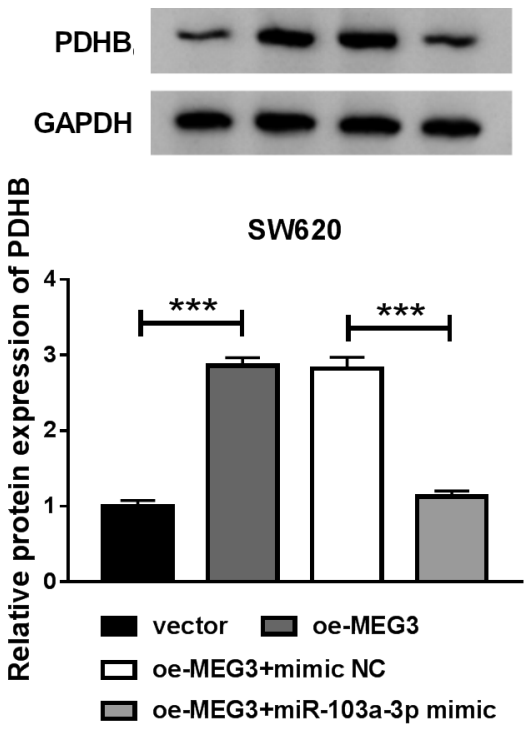

D
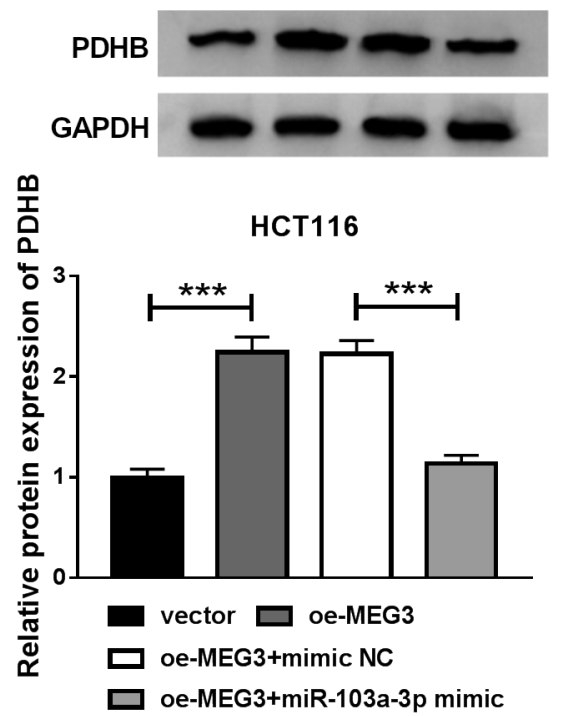

Figure 6. Validating the relationship among MEG3, miR-103a-3p, and PDHB in CC cells. RT-qPCR and western blotting detected the relative PDHB expression on $(A, C)$ mRNA level and $(B, D)$ protein level in SW620 and HCT116 cells transfected with vector, oe-MEG3, and oe-MEG3 combined with miR-103a-3p mimic or mimic NC. 
ated in nude mice with SW620 cells transplantation, and tumor growth was restrained after oe-MEG3 pre-transfection, as indicated by lowered tumor volume and weight (Figures 7A, 7B). In the oe-MEG3 group, MEG3 expression was highly expressed in xenograft tumor tissues, paralleled with miR-103a-3p downregulation and PDHB upregulation (Figure 7C). Additionally, protein levels of GRP78, ATF6, CHOP, caspase- 3 , and caspase- 9 were elevated in mice tumors, as well (Figures 7D). These data demonstrated that MEG3 also suppressed CC in vivo by inhibiting cell growth and promoting ER stress via regulation of miR-103a-3p and PDHB.

\section{Discussion}

To cope with persistent ER stress, UPR was insufficiently triggered to restore protein homeostasis, later committing cells to apoptosis. Thus, the understanding of the potential molecular mechanism of ER stress in tumorigenesis and the development of cancer cells could be critical. In CC, a few of IncRNAs had been declared to regulate ER stress or be regulated by ER stress. For example, thapsigargin induced ER stress and migration of HT29 and HCT116 cells probably through increasing lncRNA MALAT1 and activating IRE1/ XBP1 and PERK/eIF2a/ATF4 (two of three pathways of UPR) signaling pathways [23]. LncRNA LUCRC contributed to CC cell proliferation, migration, and invasion in HCT116 cells and tumorigenesis in xenografts by regulation of genes involved in ER stress including BIP [24]. Since the involvement of ER stress in MEG3-induced apoptosis had been discovered in different malignant tumor cells [25-28], it seemed uncovered in CC prior to this study. Therefore, we aimed to explore the relationship between MEG3 and ER stress-mediated apoptosis and the UPR pathway in CC cells, as well as proliferation and invasion.

Here, we supported the downregulation of MEG3 in human CC tumor tissues and cells, which had been well documented in previous studies [29-31]. Besides, decreased MEG3 was also observed in the serum of CC patients, and this downregulation was associated with larger tumor size, advanced clinical stage, and poor prognosis [32]. Low MEG3 in tumor tissue was also correlated with shorter overall survival and disease-free survival of CC patients [30, 33], as well as low histological grade, deep tumor invasion, and advanced tumor node metastasis (TNM) stage [31]. Thus, MEG3 was proposed to be one of the biomarkers for CC staging and progression [15]. Moreover, its expression was even inhibited in oxaliplatin-resistant CC patients [33], suggesting MEG3 as a potential indicator to identify oxaliplatin-responding patients [34]. Interestingly, startling data was reported by Liu et al. [14] regarding the upregulation of MEG3 together with lncRNAs $91 \mathrm{H}$ and PVT-1 in CC patients' plasma. The combination of these three lncRNAs could be a better approach to the detection of early-stage
A

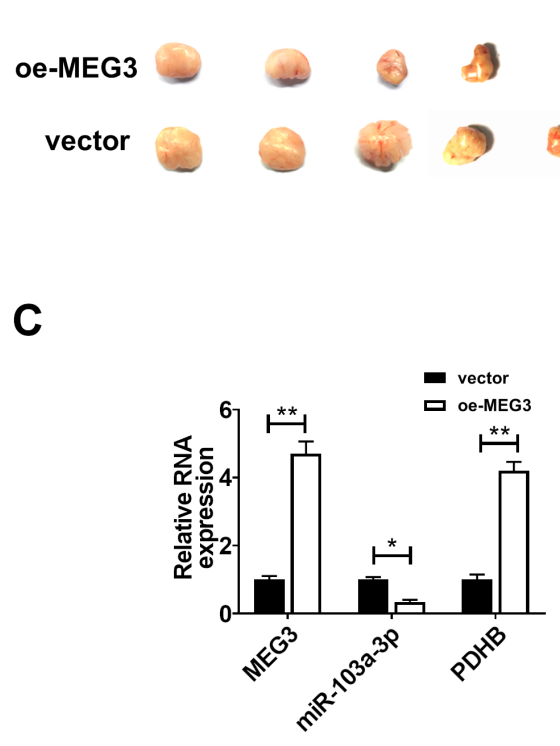

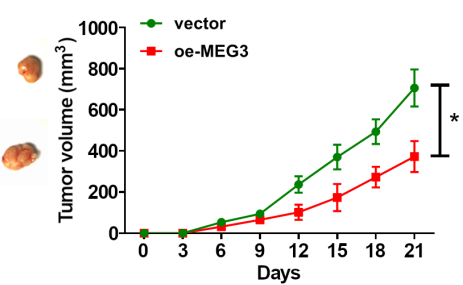

D

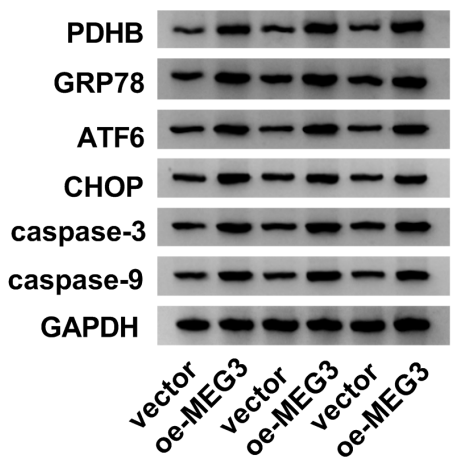

B
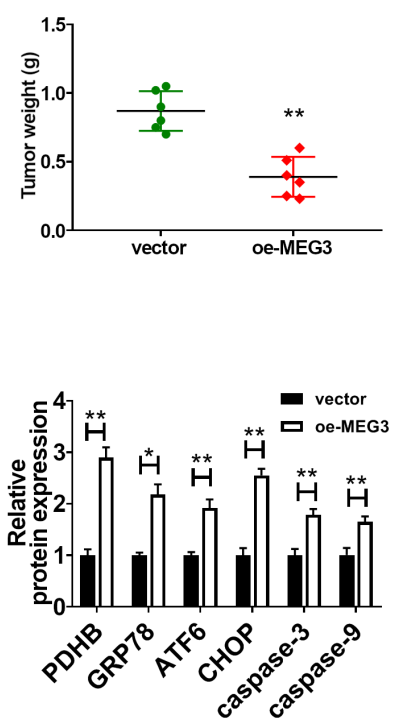

Figure 7. Investigating the role of MEG3 in cell growth and ER stress of CC cells in vivo. A-D) SW620 cells stably transfected with oe-MEG3 were subcutaneously transplanted into nude mice $(n=6)$. (A) Tumor volume was calculated every three days after transplantation. (B) Tumor weight was measured on the last day of the xenograft experiment. (C) RT-qPCR detected the relative RNA expression of MEG3, miR-103a-3p, and PDHB in xenograft tumor tissues. (D) Western blotting tested the relative protein expression of PDHB, GRP78, ATF6, CHOP, caspase-3, and caspase-9 in three randomly selected xenograft tumor tissues. ${ }^{*} \mathrm{p}<0.05$ and ${ }^{* *} \mathrm{p}<0.01$. 
CC. We thought that conflicting findings might be attributed to the difference in control plasma samples, as high MEG3 finding was compared to a mixture of healthy people and patients with benign gastrointestinal inflammation diseases [14], whereas low MEG3 was normalized to healthy people only [32].

In CC, MEG3 had been illustrated to be implicated in malignant cell functions [32], glycolysis [35], and chemoresistance [34] of CC cells. Next, we verified an anti-tumor role of MEG3 in CC cells by inhibiting cell viability, colony formation ability, cell cycle progression and invasion, and promoting ER stress. The inhibitory effect of MEG3 on proliferation and migration and its promoting effect on apoptosis were consistent with other researches [29-32]. However, the underlying mechanism of MEG3 inducing apoptosis was left to be detected. In this study, we noticed that the UPR response was accompanied by the apoptosis of CC cells, hinting an ER stress-induced apoptosis via the ATF6 pathway and caspase-3/9 pathway. In mechanism, a MEG3/miR-103a-3p/PDHB ceRNA axis was further identified. miR-103a-3p was the most stable reference miRNA in tumor tissues of several cancers [36-38], including CC [38]. Furthermore, this miRNA in tissue was one essential member of a miRNA-based classifier in prognosis and prediction of stage II CC both in Asian patients and white patients [19, 39]. A panel of 7-miRNA signature in plasma including miR-103a-3p was established for CC diagnosis according to a four-stage (I, II, III, and IV) study [18]. However, its cellular functions had been obscured till this present study. Silencing miR-103a-3p suppressed cell viability, colony formation ability, cell cycle progression, and invasion, but enhanced ER stress-induced apoptosis. Notably, this finding might be the first evidence demonstrating the association between miR-103a-3p and ER stress.

Oncogenic activities of PDHB were deciphered via transfection in glioma cells [40], melanoma cells [41], ovarian cancer cells [42], nasopharyngeal carcinoma [43], by augmenting proliferation, migration, invasion, and glycolysis, as well as inhibiting apoptosis. In CC, this investigation revealed that $\mathrm{PDHB}$ was lowly expressed in $\mathrm{CC}$ tissues and cells and that the PDHB downregulation contributed to cell viability, colony formation ability, cell cycle progression, and invasion of SW960 and HCT116 cells; in turn, restoring PDHB could abolish oncogenic effect on proliferation, invasion, and glycolysis in SW620 and HT29 cells [44].

In conclusion, this study showed that MEG3 and PDHB were downregulated in human CC tissues and cells, accompanied by the miR-103a-3p upregulation. The anti-tumor roles of MEG3 overexpression and miR-103a-3p knockdown were verified in CC progression by regulating cell proliferation, invasion, and ER stress via the MEG3/miR-103a-3p/ PDHB ceRNA pathway.

Supplementary information is available in the online version of the paper.

\section{References}

[1] SIWECKA N, ROZPEDEK W, PYTEL D, WAWRZYNKIEWICZ A, DZIKI A et al. Dual role of Endoplasmic Reticulum Stress-Mediated Unfolded Protein Response Signaling Pathway in Carcinogenesis. Int J Mol Sci 2019; 20: 4354. https://doi.org/10.3390/ijms20184354

[2] KIM C, KIM B. Anti-Cancer Natural Products and Their Bioactive Compounds Inducing ER Stress-Mediated Apoptosis: A Review. Nutrients 2018; 10: 1021. https://doi.org/10.3390/ nu10081021

[3] LIMIA CM, SAUZAY C, URRA H, HETZ C, CHEVET E et al. Emerging Roles of the Endoplasmic Reticulum Associated Unfolded Protein Response in Cancer Cell Migration and Invasion. Cancers (Basel) 2019; 11: 631. https://doi. org/10.3390/cancers11050631

[4] LIN Y, JIANG M, CHEN W, ZHAO T, WEI Y. Cancer and ER stress: Mutual crosstalk between autophagy, oxidative stress and inflammatory response. Biomed Pharmacother 2019; 118: 109249. https://doi.org/10.1016/j.biopha.2019.109249

[5] CHEVET E, HETZ C, SAMALI A. Endoplasmic reticulum stress-activated cell reprogramming in oncogenesis. Cancer Discov 2015; 5: 586-597. https://doi.org/10.1158/2159-8290. CD-14-1490

[6] LIMONTA P, MORETTI RM, MARZAGALLI M, FONTANA F, RAIMONDI $M$ et al. Role of Endoplasmic Reticulum Stress in the Anticancer Activity of Natural Compounds. Int J Mol Sci 2019; 20: 961. https://doi.org/10.3390/ ijms20040961

[7] MOKARRAM P, ALBOKASHY M, ZARGHOONI M, MOOSAVI MA, SEPEHRI $Z$ et al. New frontiers in the treatment of colorectal cancer: Autophagy and the unfolded protein response as promising targets. Autophagy 2017; 13: 781-819. https://doi.org/10.1080/15548627.2017.1290751

[8] COLEMAN OI, HALLER D. ER Stress and the UPR in Shaping Intestinal Tissue Homeostasis and Immunity. Front Immunol 2019; 10: 2825. https://doi.org/10.3389/fimmu.2019.02825

[9] BRAY F, FERLAY J, SOERJOMATARAM I, SIEGEL RL, TORRE LA et al. Global cancer statistics 2018: GLOBOCAN estimates of incidence and mortality worldwide for 36 cancers in 185 countries. CA Cancer J Clin 2018; 68: 394-424. https://doi.org/10.3322/caac. 21492

[10] ARNOLD M, SIERRA MS, LAVERSANNE M, SOERJOMATARAM I, JEMAL A et al. Global patterns and trends in colorectal cancer incidence and mortality. Gut 2017; 66: 683-691. https://doi.org/10.1136/gutjnl-2015-310912

[11] SIEGEL RL, MILLER KD, JEMAL A. Cancer statistics, 2019. CA Cancer J Clin 2019; 69: 7-34. https://doi.org/10.3322/ caac. 21551

[12] WANG L, CHO KB, LI Y, TAO G, XIE Z et al. Long Noncoding RNA (lncRNA)-Mediated Competing Endogenous RNA Networks Provide Novel Potential Biomarkers and Therapeutic Targets for Colorectal Cancer. Int J Mol Sci 2019; 20: 5758. https://doi.org/10.3390/ijms20225758

[13] GALAMB O, BARTAK BK, KALMAR A, NAGY ZB, SZIGETI KA et al. Diagnostic and prognostic potential of tissue and circulating long non-coding RNAs in colorectal tumors. World J Gastroenterol 2019; 25: 5026-5048. https:// doi.org/10.3748/wjg.v25.i34.5026 
[14] LIU H, YE D, CHEN A, TAN D, ZHANG W et al. A pilot study of new promising non-coding RNA diagnostic biomarkers for early-stage colorectal cancers. Clin Chem Lab Med 2019; 57: 1073-1083. https://doi.org/10.1515/cclm2019-0052

[15] WU M, LI W, HUANG F, SUN J, LI KP et al. Comprehensive Analysis of the Expression Profiles of Long Non-Coding RNAs with Associated ceRNA Network Involved in the Colon Cancer Staging and Progression. Sci Rep 2019; 9: 16910. https://doi.org/10.1038/s41598-019-52883-2

[16] TANG XJ, WANG W, HANN SS. Interactions among lncRNAs, miRNAs and mRNA in colorectal cancer. Biochimie 2019; 163: 58-72. https://doi.org/10.1016/j.biochi.2019.05.010

[17] DING L, LAN Z, XIONG X, AO H, FENG Y et al. The Dual Role of MicroRNAs in Colorectal Cancer Progression. Int J Mol Sci 2018; 19: 2791. https://doi.org/10.3390/ ijms 19092791

[18] ZHANG H, ZHU M, SHAN X, ZHOU X, WANG T et al. A panel of seven-miRNA signature in plasma as potential biomarker for colorectal cancer diagnosis. Gene 2019; 687: 246-254. https://doi.org/10.1016/j.gene.2018.11.055

[19] ZHANG JX, SONG W, CHEN ZH, WEI JH, LIAO YJ et al. Prognostic and predictive value of a microRNA signature in stage II colon cancer: a microRNA expression analysis. Lancet Oncol 2013; 14: 1295-1306. https://doi.org/10.1016/ S1470-2045(13)70491-1

[20] FUHR L, EL-ATHMAN R, SCRIMA R, CELA O, CARBONE A et al. The Circadian Clock Regulates Metabolic Phenotype Rewiring Via HKDC1 and Modulates Tumor Progression and Drug Response in Colorectal Cancer. EBioMedicine 2018; 33: 105-121. https://doi.org/10.1016/j.ebiom.2018.07.002

[21] BAI J, XU J, ZHAO J, ZHANG R. IncRNA SNHG1 cooperated with miR-497/miR-195-5p to modify epithelial-mesenchymal transition underlying colorectal cancer exacerbation. J Cell Physiol 2020; 235: 1453-1468. https://doi.org/10.1002/ jcp. 29065

[22] ZHANG R, CHUNG Y, KIM HS, KIM DH, KIM HS et al. 20-O-(beta-D-glucopyranosyl)-20(S)-protopanaxadiol induces apoptosis via induction of endoplasmic reticulum stress in human colon cancer cells. Oncol Rep 2013; 29: 1365-1370. https://doi.org/10.3892/or.2013.2270

[23] JIANG X, LI D, WANG G, LIU J, SU X et al. Thapsigargin promotes colorectal cancer cell migration through upregulation of lncRNA MALAT1. Oncol Rep 2020; 43: 1245-1255. https://doi.org/10.3892/or.2020.7502

[24] TANG GH, CHEN X, DING JC, DU J, LIN XT et al. LncRNA LUCRC Regulates Colorectal Cancer Cell Growth and Tumorigenesis by Targeting Endoplasmic Reticulum Stress Response. Front Genet 2020; 10: 1409. https://doi. org/10.3389/fgene.2019.01409

[25] PAN X, CAO YM, LIU JH, DING J, XIE XY et al. MEG3 Induces Cervical Carcinoma Cells' Apoptosis Through Endoplasmic Reticulum Stress by miR-7-5p/STC1 Axis. Cancer Biother Radiopharm 2020. https://doi.org/10.1089/ cbr.2019.3344
[26] ZHANG Y, WU J, JING H, HUANG G, SUN Z et al. Long noncoding RNA MEG3 inhibits breast cancer growth via upregulating endoplasmic reticulum stress and activating NF-kappaB and p53. J Cell Biochem 2019; 120: 6789-6797. https://doi.org/10.1002/jcb.27982

[27] HUANG ZL, CHEN RP, ZHOU XT, ZHAN HL, HU MM et al. Long non-coding RNA MEG3 induces cell apoptosis in esophageal cancer through endoplasmic reticulum stress. Oncol Rep 2017; 37: 3093-3099. https://doi.org/10.3892/ or.2017.5568

[28] CHEN RP, HUANG ZL, LIU LX, XIANG MQ, LI GP et al. Involvement of endoplasmic reticulum stress and p53 in lncRNA MEG3-induced human hepatoma HepG2 cell apoptosis. Oncol Rep 2016; 36: 1649-1657. https://doi.org/10.3892/ or.2016.4919

[29] WU X, LI J, REN Y, ZUO Z, NI S et al. MEG3 can affect the proliferation and migration of colorectal cancer cells through regulating miR-376/PRKD1 axis. Am J Transl Res 2019; 11: 5740-5751.

[30] ZHU Y, CHEN P, GAO Y, TA N, ZHANG Y et al. MEG3 Activated by Vitamin D Inhibits Colorectal Cancer Cells Proliferation and Migration via Regulating Clusterin. EBioMedicine 2018; 30: 148-157. https://doi.org/10.1016/j.ebiom.2018.03.032

[31] YIN DD, LIU ZJ, ZHANG E, KONG R, ZHANG ZH et al. Decreased expression of long noncoding RNA MEG3 affects cell proliferation and predicts a poor prognosis in patients with colorectal cancer. Tumour Biol 2015; 36: 4851-4859. https://doi.org/10.1007/s13277-015-3139-2

[32] WANG W, XIE Y, CHEN F, LIU X, ZHONG LL et al. LncRNA MEG3 acts a biomarker and regulates cell functions by targeting ADAR1 in colorectal cancer. World J Gastroenterol 2019; 25: 3972-3984. https://doi.org/10.3748/wjg.v25.i29.3972

[33] WANG H, LI H, ZHANG L, YANG D. Overexpression of MEG3 sensitizes colorectal cancer cells to oxaliplatin through regulation of miR-141/PDCD4 axis. World J Gastroenterol 2019; 25: 3972-3984. https://doi.org/10.3748/wjg. v25.i29.3972

[34] LI L, SHANG J, ZHANG Y, LIU S, PENG Y et al. MEG3 is a prognostic factor for CRC and promotes chemosensitivity by enhancing oxaliplatin-induced cell apoptosis. Oncol Rep 2017; 38: 1383-1392. https://doi.org/10.3892/or.2017.5828

[35] ZUO S, WU L, WANG Y, YUAN X. Long Non-coding RNA MEG3 Activated by Vitamin D Suppresses Glycolysis in Colorectal Cancer via Promoting c-Myc Degradation. Front Oncol 2020; 10: 274. https://doi.org/10.3389/ fonc.2020.00274

[36] PELTIER HJ, LATHAM GJ. Normalization of microRNA expression levels in quantitative RT-PCR assays: identification of suitable reference RNA targets in normal and cancerous human solid tissues. RNA 2008; 14: 844-852. https://doi. org/10.1261/rna.939908

[37] WOTSCHOFSKY Z, MEYER HA, JUNG M, FENDLER A, WAGNER I et al. Reference genes for the relative quantification of microRNAs in renal cell carcinomas and their metastases. Anal Biochem 2011; 417: 233-241. https://doi. org/10.1016/j.ab.2011.06.009 
[38] BOISEN MK, DEHLENDORFF C, LINNEMANN D, SCHULTZ NA, JENSEN BV et al. MicroRNA Expression in Formalin-fixed Paraffin-embedded Cancer Tissue: Identifying Reference MicroRNAs and Variability. BMC Cancer 2015; 15: 1024. https://doi.org/10.1186/s12885-015-2030-2

[39] CARITG O, NAVARRO A, MORENO I, MARTINEZRODENAS F, CORDEIRO A et al. Identifying High-Risk Stage II Colon Cancer Patients: A Three-MicroRNABased Score as a Prognostic Biomarker. Clin Colorectal Cancer 2016; 15: e175-e182. https://doi.org/10.1016/j. clcc.2016.04.008

[40] XU DX, GUO JJ, ZHU GY, WU HJ, ZHANG QS et al. MiR-363-3p modulates cell growth and invasion in glioma by directly targeting pyruvate dehydrogenase B. Eur Rev Med Pharmacol Sci 2018; 22: 5230-5239. https://doi. org/10.26355/eurrev_201808_15721
[41] WEI S, MA W. MiR-370 functions as oncogene in melanoma by direct targeting pyruvate dehydrogenase B. Biomed Pharmacother 2017; 90: 278-286. https://doi.org/10.1016/j. biopha.2017.03.068

[42] XIAOHONG Z, LICHUN F, NA X, KEJIAN Z, XIAOLAN X et al. MiR-203 promotes the growth and migration of ovarian cancer cells by enhancing glycolytic pathway. Tumour Biol 2016; 37: 14989-14997. https://doi.org/10.1007/s13277-016$5415-1$

[43] TANG H, LUO X, LI J, ZHOU Y, LI Y et al. Pyruvate dehydrogenase $\mathrm{B}$ promoted the growth and migration of the nasopharyngeal carcinoma cells. Tumour Biol 2016; 37: 10563-10569. https://doi.org/10.1007/s13277-016-4922-4

[44] ZHU Y, WU G, YAN W, ZHAN H, SUN P. miR-146b-5p regulates cell growth, invasion, and metabolism by targeting PDHB in colorectal cancer. Am J Cancer Res 2017; 7: $1136-1150$. 


\section{LncRNA MEG3 promotes endoplasmic reticulum stress and suppresses proliferation and invasion of colorectal carcinoma cells through the MEG3/ miR-103a-3p/PDHB ceRNA pathway}

Guoqin WANG ${ }^{1, *}$, Qiuwen $\mathrm{YE}^{2, *}$, Shujuan NING ${ }^{3}$, Zhuangqing YANG ${ }^{4}$, Yunlan $\mathrm{CHEN}^{5}$, Lijuan ZHANG ${ }^{6}$, Youguang $\mathrm{HUANG}^{7}$, Fujia XIE ${ }^{8}$ Xi $\mathrm{CHENG}^{8}$, Junlin $\mathrm{CHI}^{8}$, Yi $\mathrm{LEI}^{8}$, Rong GUO , Jiangqiong $\mathrm{HAN}^{10, *}$

\section{Supplementary Information}

Supplementary Table S1. The dose of nucleotides and transfection reagent used in this study.

\begin{tabular}{|c|c|c|c|}
\hline & Component & 96-well plate & 6-well plate \\
\hline \multirow[t]{2}{*}{ A solution } & Opti-MEM medium & $5 \mu \mathrm{L}$ & $125 \mu \mathrm{L}$ \\
\hline & Lipofectamine 3000 reagent & $0.15 \mu \mathrm{L}$ & $3.75 \mu \mathrm{L}$ \\
\hline \multicolumn{4}{|c|}{ Standing for $5 \mathrm{~min}$ at room temperature. } \\
\hline \multirow{3}{*}{ B solution } & Opti-MEM medium & $5 \mu \mathrm{L}$ & $125 \mu \mathrm{L}$ \\
\hline & Nucleotides & $0.1 \mu \mathrm{g}$ or $2 \mathrm{nM}$ & $2.5 \mu \mathrm{g}$ \\
\hline & $\begin{array}{l}\text { P3000 reagent (Except } \\
\text { siRNA) }\end{array}$ & $0.2 \mu \mathrm{L}$ & $5 \mu \mathrm{L}$ \\
\hline \multicolumn{4}{|c|}{ Standing for $5 \mathrm{~min}$ at room temperature. } \\
\hline
\end{tabular}


A
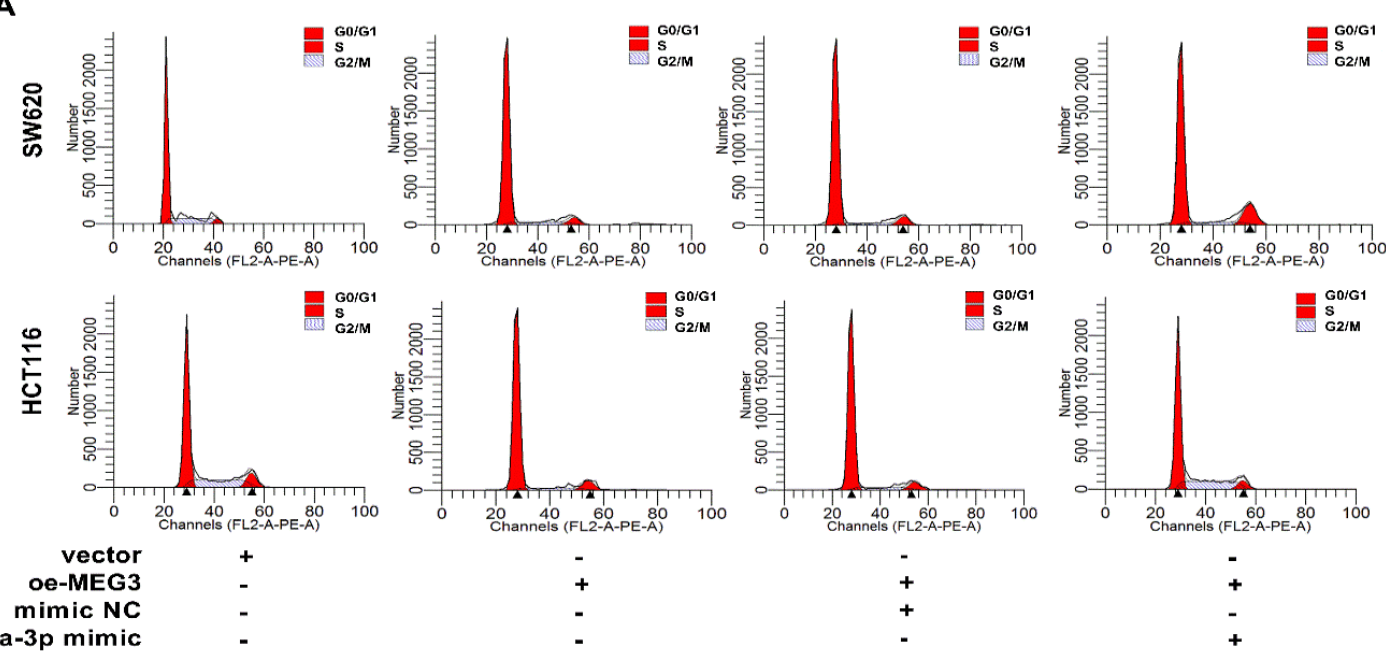

miR-103a-3p mimic

$\begin{aligned} \text { vector } & + \\ \text { oe-MEG3 } & - \\ \text { mimic NC } & - \\ -3 p \text { mimic } & -\end{aligned}$

B
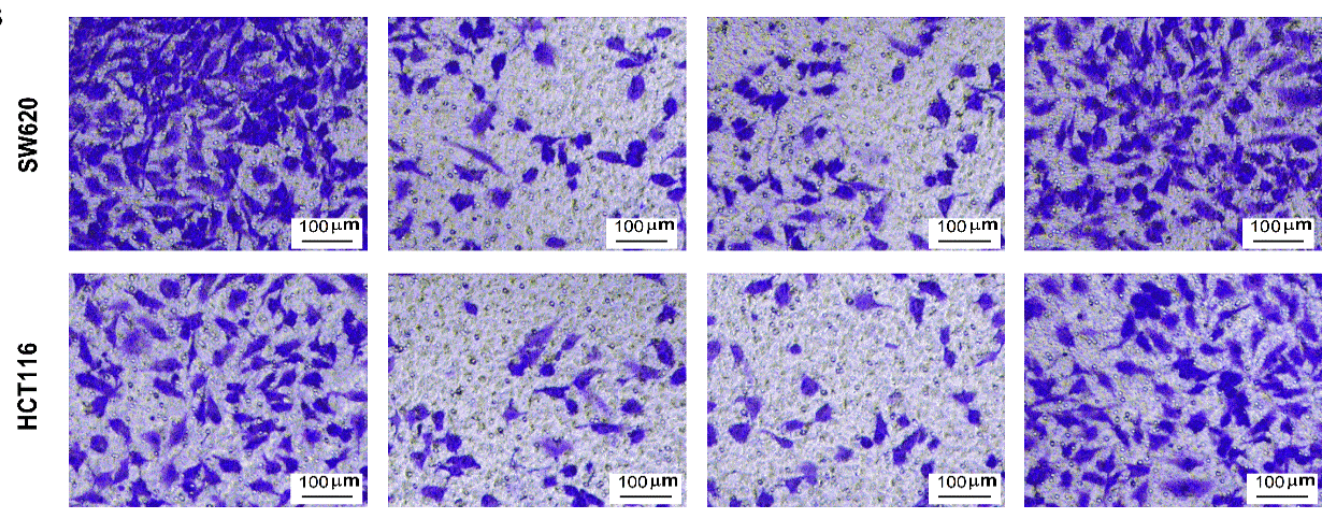

$\begin{aligned} \text { vector } & + \\ \text { oe-MEG3 } & - \\ \text { mimic NC } & -\end{aligned}$

miR-103a-3

\section{C}
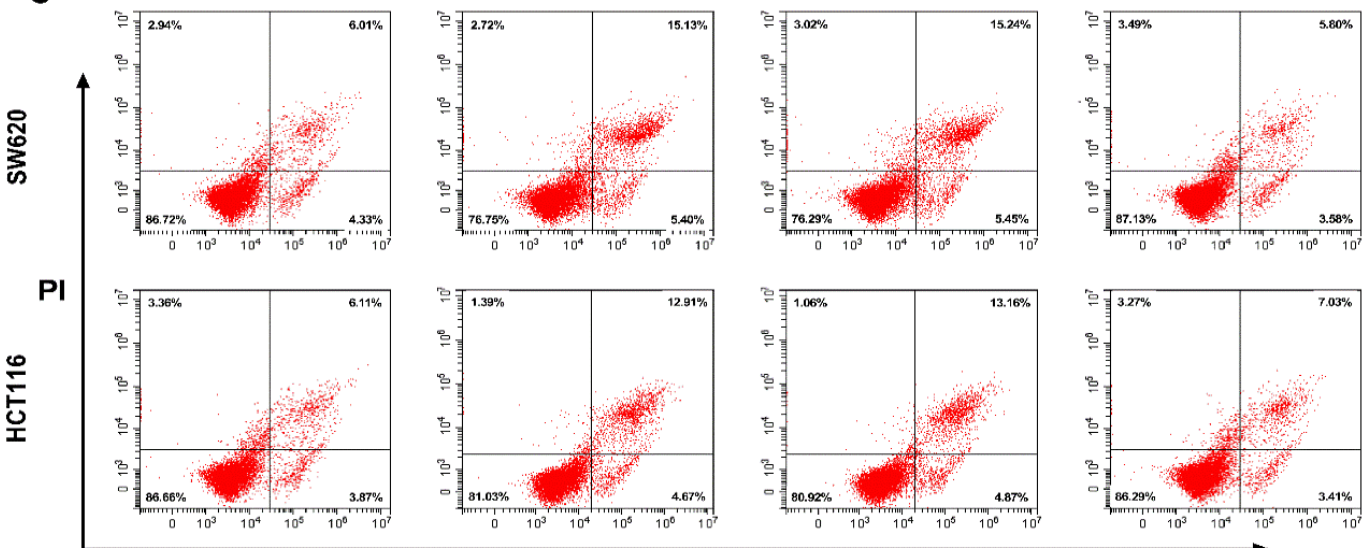

Annexin V-FITC

$\begin{aligned} \text { vector } & + \\ \text { oe-MEG3 } & - \\ \text { mimic NC } & - \\ \text { miR-103a-3p mimic } & -\end{aligned}$

Supplementary Figure S1. The images of transwell assay, flow cytometric cell cycle analysis, and apoptosis assay in SW620 and HCT116 cells that underwent transfections of MEG3 overexpressing vector (oe-MEG3), empty vector (vector), mimics of miR-103a-3p, or its negative control. 
A
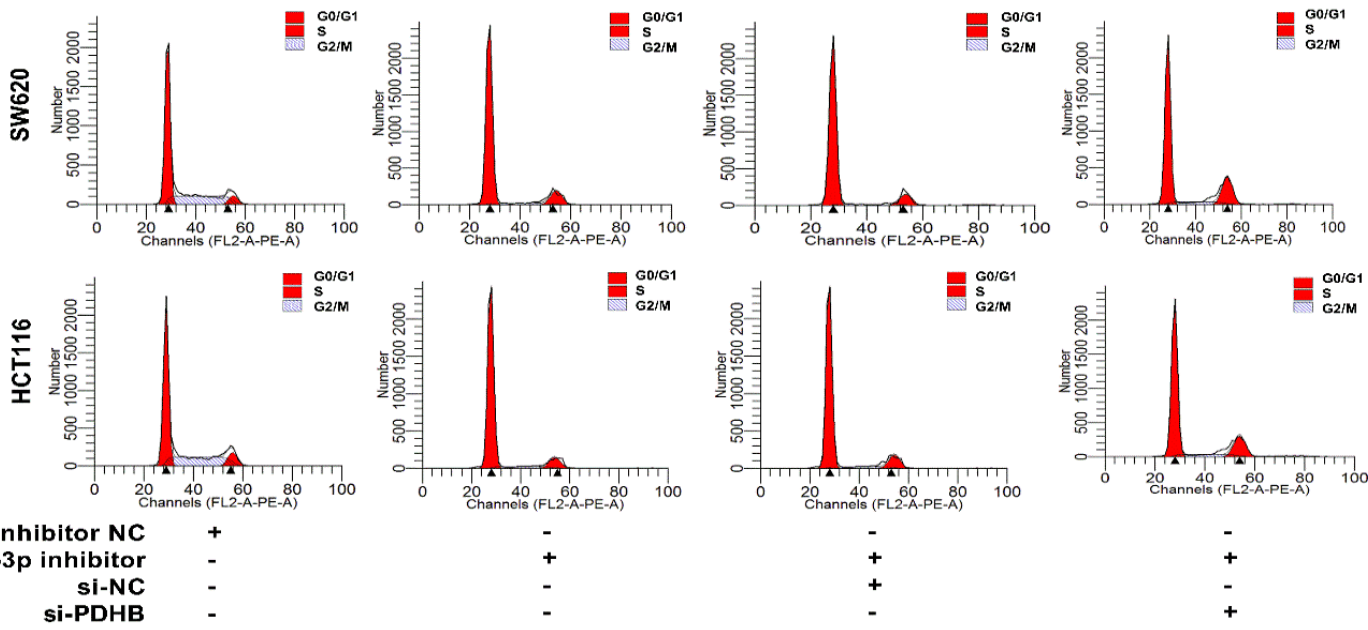

B

$\begin{aligned} \text { inhibitor NC } & + \\ -3 p \text { inhibitor } & - \\ \text { si-NC } & - \\ \text { si-PDHB } & -\end{aligned}$
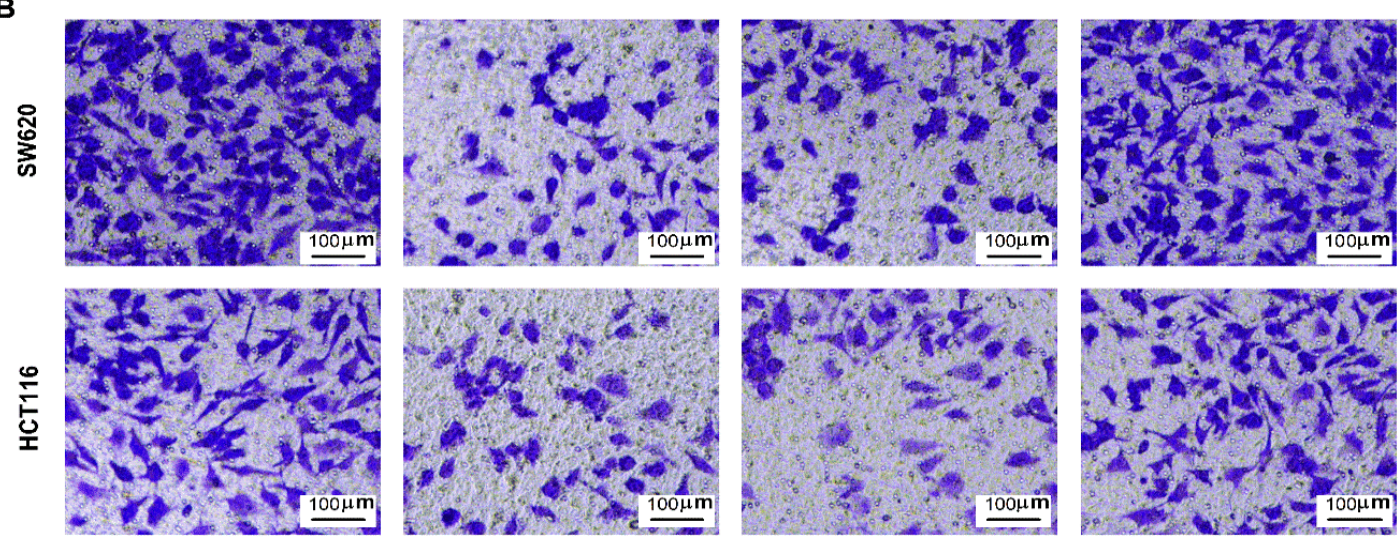

miR-103a-3p inhibitor si-NC

C
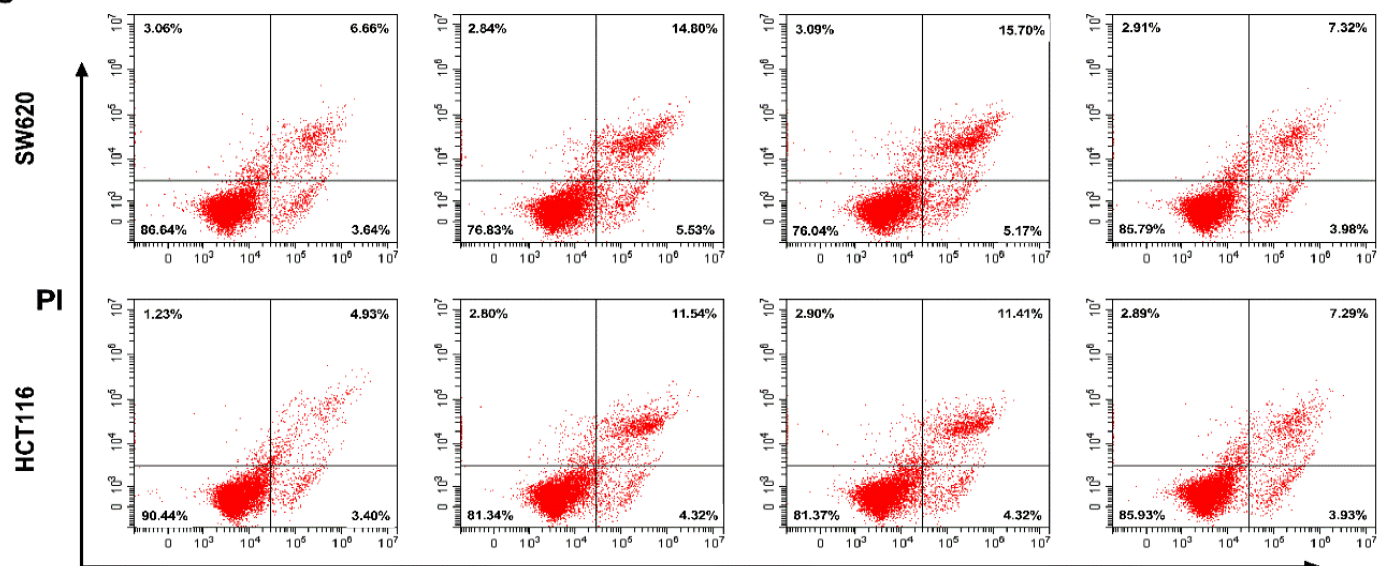

Annexin V-FITC

inhibitor NC
miR-103a-3p inhibitor
si-NC
si-PDHB

Supplementary Figure S2. The images of transwell assay, flow cytometric cell cycle analysis, and apoptosis assay in SW620 and HCT116 cells that underwent transfections of miR-103a-3p inhibitor, inhibitor negative control, siRNAs target PDHB, or the negative control (si-PDHB or si-NC). 